\title{
Propagation of satellite orbit modelling deficiencies into the global GNSS solutions - simulation-based study
}

\author{
Maciej Kalarus, Rolf Dach, Arturo Villiger, and Adrian Jaeggi
}

Astronomical Institute, University of Bern 


\section{Abstract}

The Non-Gravitational Perturbations (NGP), out of which the Solar Radiation Pressure (SRP) is the largest, have a significant impact on GNSS satellite orbits. In addition to the SRP, other relevant perturbations should also be taken into account, as this may result in substantial modelling errors if underestimated. Particularly, the force model should also consider Earth's albedo in terms of the emitted and reflected radiation, as well as a physical satellite model (box-wing) with its optical and thermal properties.

GNSS satellite orbit modelling may suffer from deficiencies for various reasons (simplification of the complexity of the used model or uncertainty of the input information). The impact of such model errors on global GNSS data analyses is assessed in an error propagation study based on simulated observations. The influence of artificially introduced orbit errors on estimated parameters, e.g. Earth rotation parameters, orbit parameters (initial conditions and dynamical orbit parameters), station coordinates, station-wise troposphere parameters, as well as receiver and satellite clock corrections is investigated.

In this study a dedicated simulation environment is used to analyse the relation between results (e.g. orbits, satellite clocks, Earth orientation parameters) and certain individual shortcomings in the NGP models. In addition, apart from a commonly used epoch-wise clock estimation, the analytical models for satellite clock corrections are introduced in order to exploit the high stability of the passive $\mathrm{H}$ masers on-board the Galileo satellites. The simulation environment also allows to assess the impact of float- versus fixed-ambiguities. Finally, simulation-based analyses offer an excellent framework for more detailed validations and further refinements of the physical satellite models, which will 


\section{Motivation}

- understand how the results of precise orbit determination (POD) are affected by mismodelled non-gravitational forces acting on the satellites

- assess how the potential exploitation of the stable passive H-masers (by applying linear clock model) may influence the POD solution in a presence of mismodelled nongravitational forces 


\section{Simulation setup - observation sites, satellite constellation}

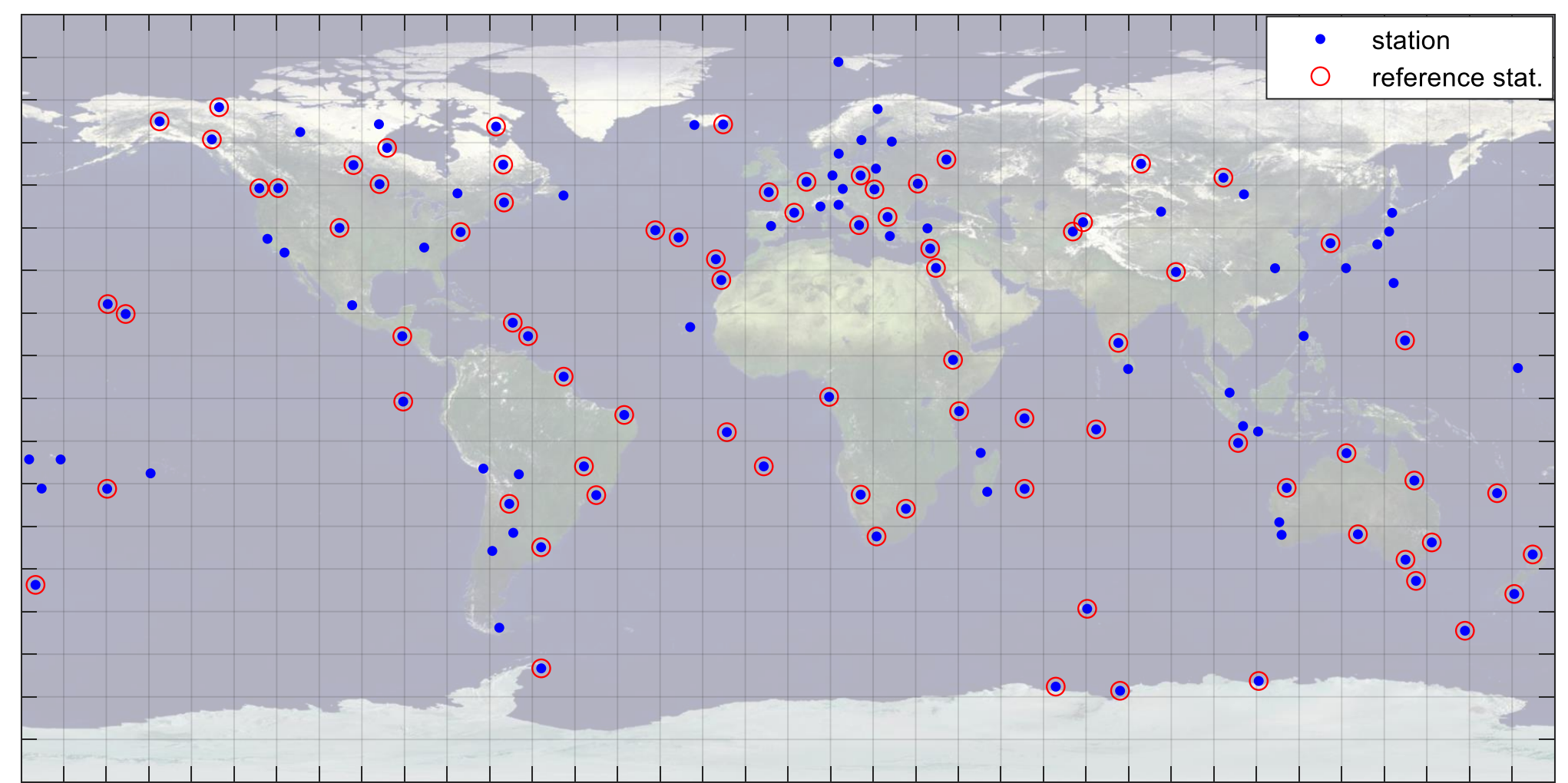

- stations contributing to the solution: 129 stations (79 reference stations)

- phase observations with $1 \mathrm{~mm}$ noise level (366 daily batches)

- Galileo constellation (as in 2020):

- 3x IOV (In Orbit Validation)

- 21x FOC (Full Operational Capability) (including 2 satellites on elliptical orbits) 


\section{Simulation setup - processing scheme (simulation)}

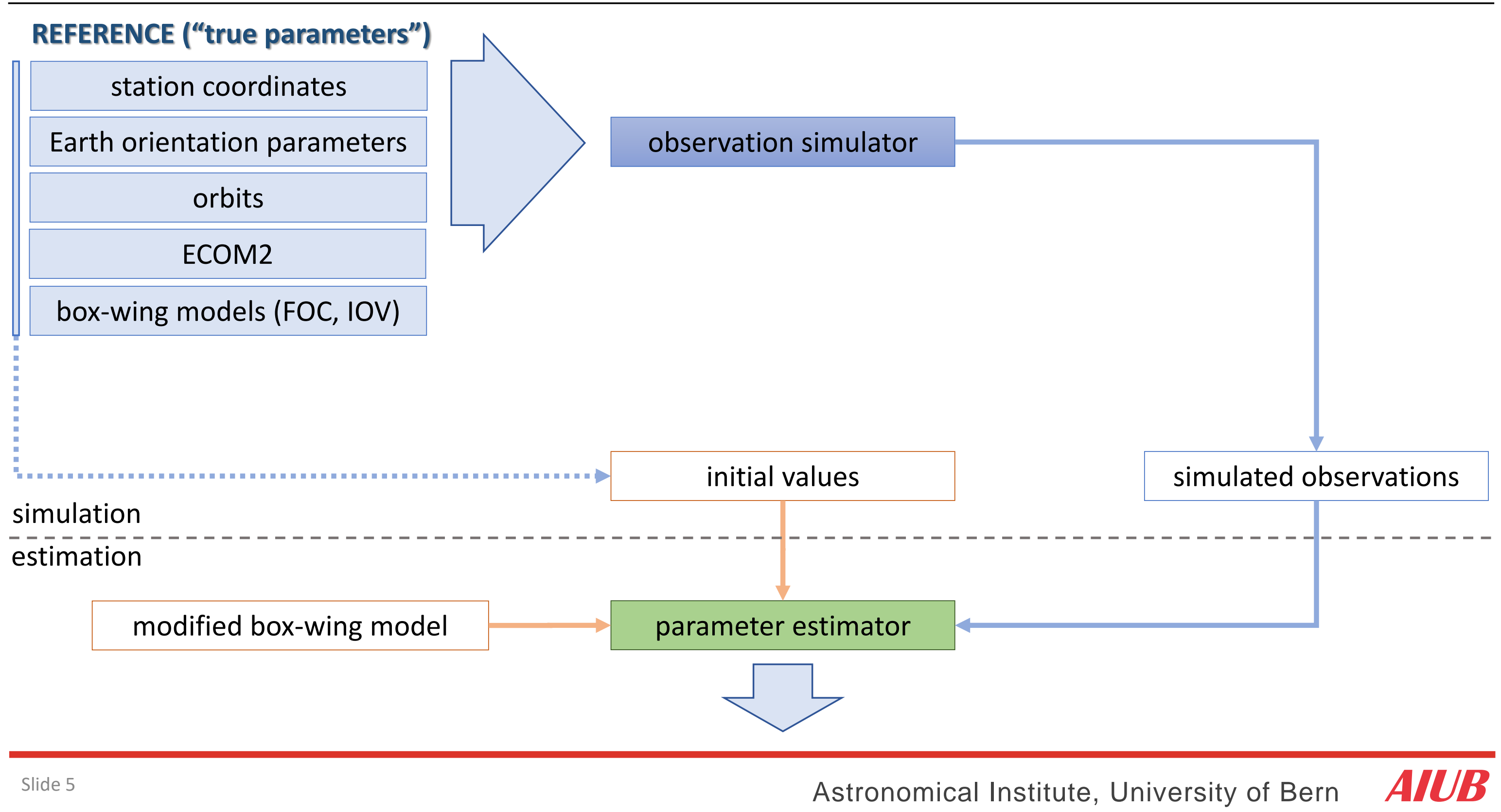




\section{Simulation setup - processing scheme (estimation)}

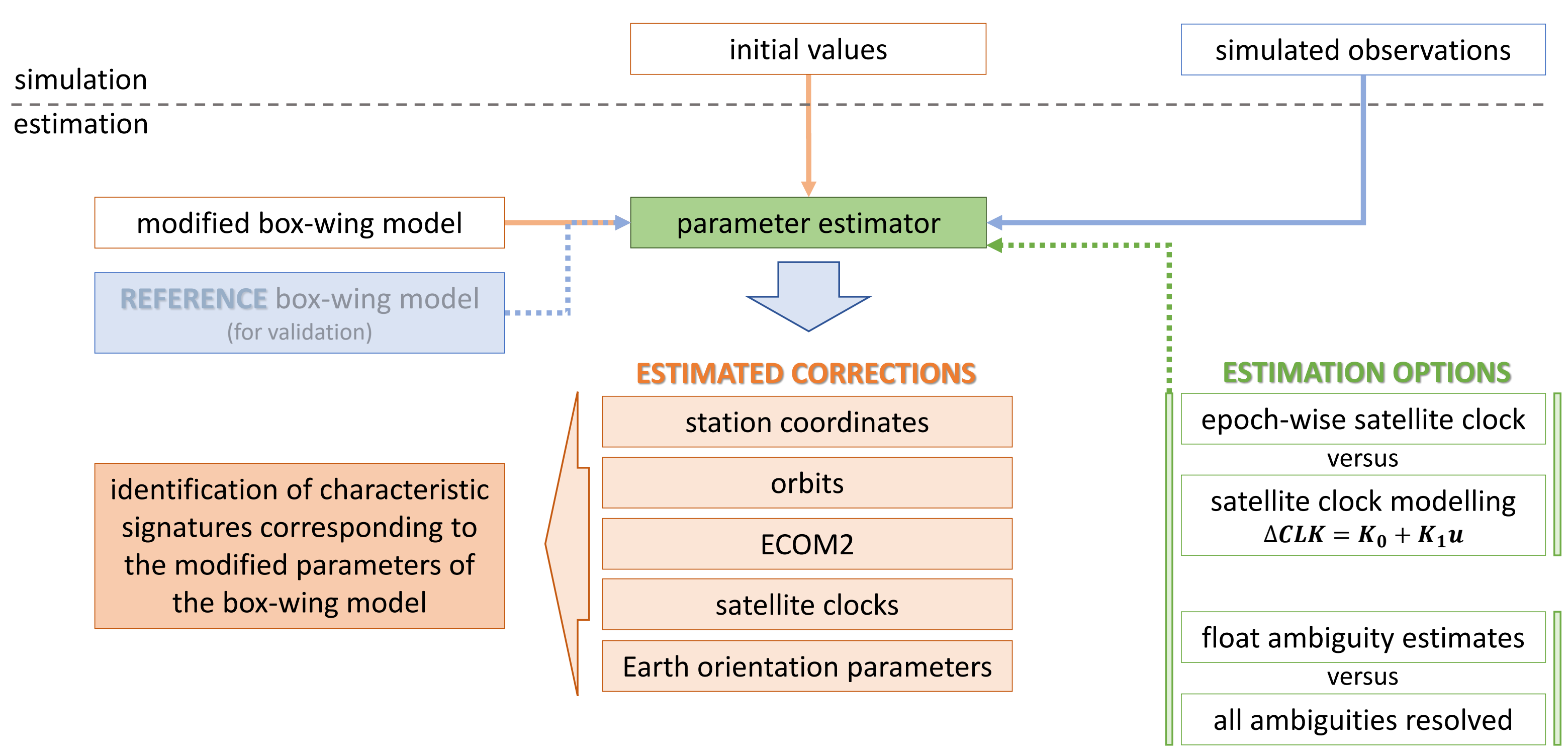




\section{Simulation setup - processing scheme (estimation details)}

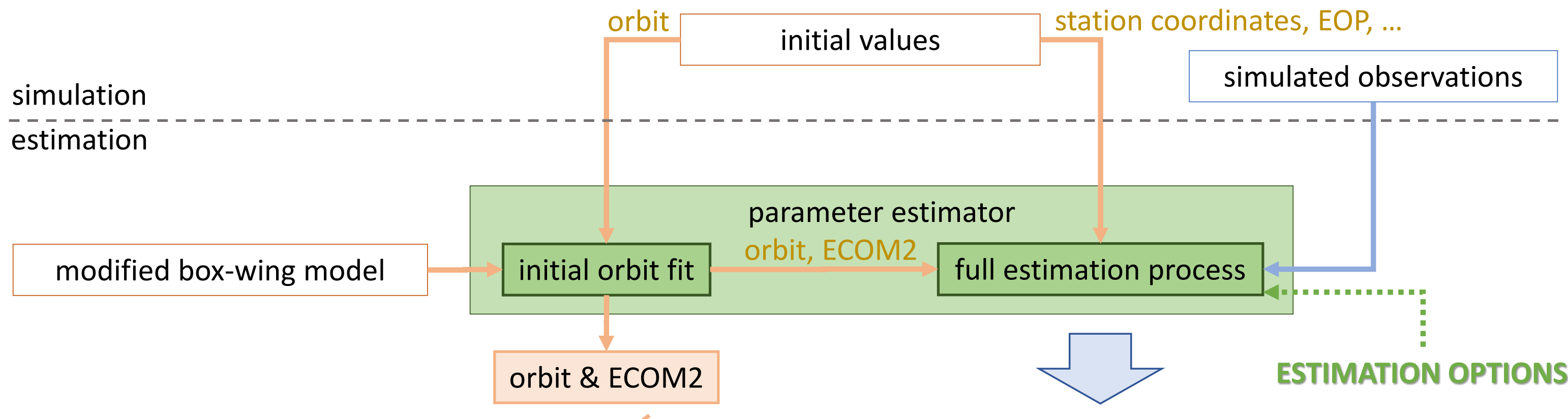

This solution indicates how much of the mismodelled non-gravitational terms can be captured by ECOM2 parameters.

\section{ESTIMATED CORRECTIONS}

This solution indicates how the mismodelled non-gravitational forces impact POD estimates.

Mismodelled NGP not absorbed by ECOM2 will affect other parameters like station coordinates, clock corrections, EOPs, etc. 


\section{Simulation setup - ECOM model used}

CODE: Center for Orbit Determination in Europe

ECOM: Empirical CODE Orbit Model

\section{ECOM2 frame:}

D : satellite-Sun direction

Y : along the solar panels

B : completes the right-handed system

\section{ECOM2 terms (empirical accelerations):}

$\mathrm{D}_{0}, \mathrm{Y}_{0}, \mathrm{~B}_{0} \quad$ : constant terms

$\mathrm{D}_{1 \mathrm{~S}}, \mathrm{~B}_{1 \mathrm{C}}, \mathrm{B}_{1 \mathrm{~S}}$ : one per revolution terms (cosine, sine)

$\mathrm{D}_{2 \mathrm{C}}, \mathrm{D}_{2 \mathrm{~S}} \quad$ : two per revolution terms (cosine, sine)

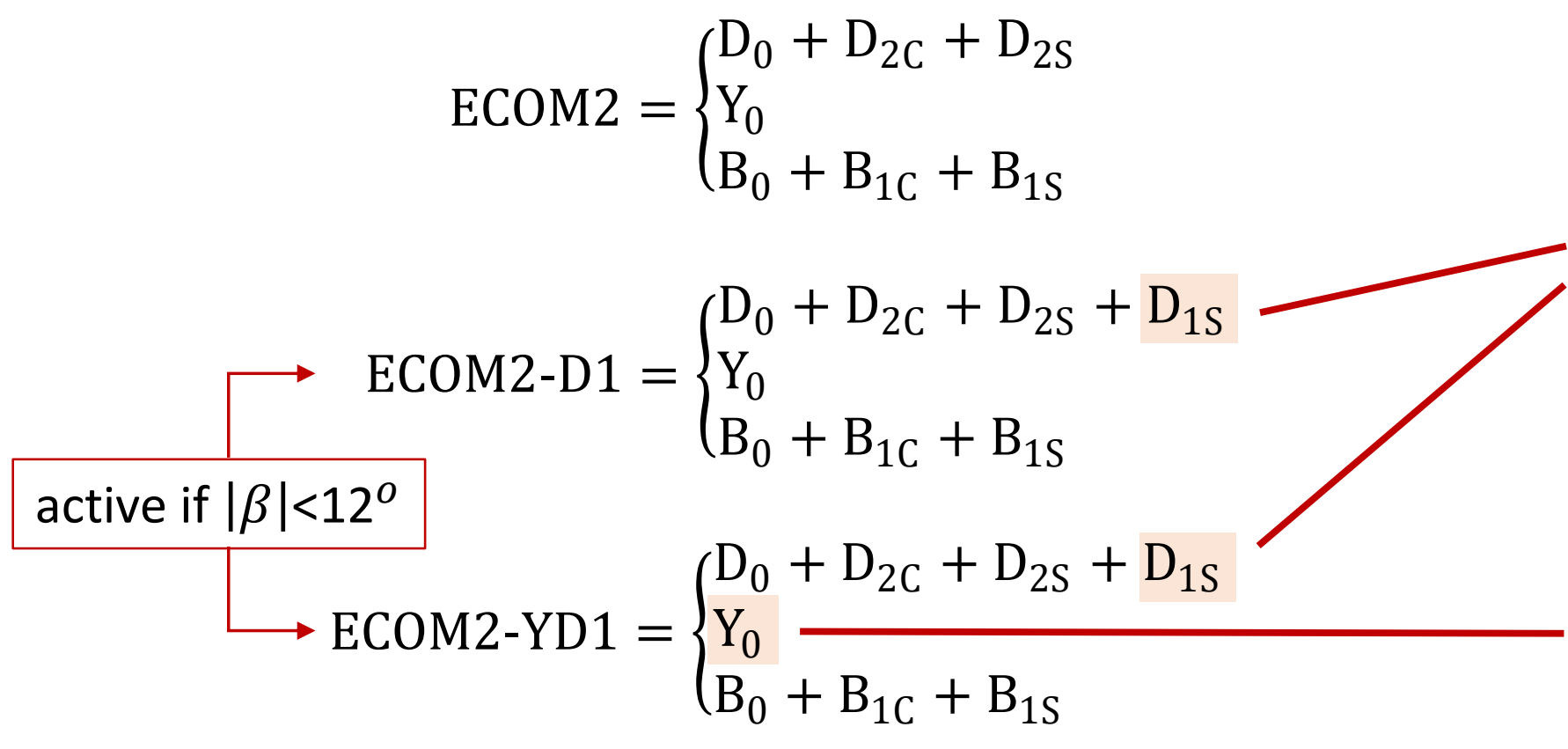

$\beta$ : elevation of the Sun with respect to the orbital plane

active in eclipses to capture thermal effects during Earth's shadow transitions

active in eclipses for the Galileo FOC satellites to capture the thermal imbalance between the $+Y$ and $-Y$ faces of these spacecraft 


\section{Simulation setup - non-gravitational perturbations acting on the satellite (example)}

Satellite box-wing model:

- size (area of each satellite box plane, area of solar panels)

Solar irradiance

- mass

- thermal properties

- surface optical properties

- diffuse reflection coefficients

- specular reflection coefficients

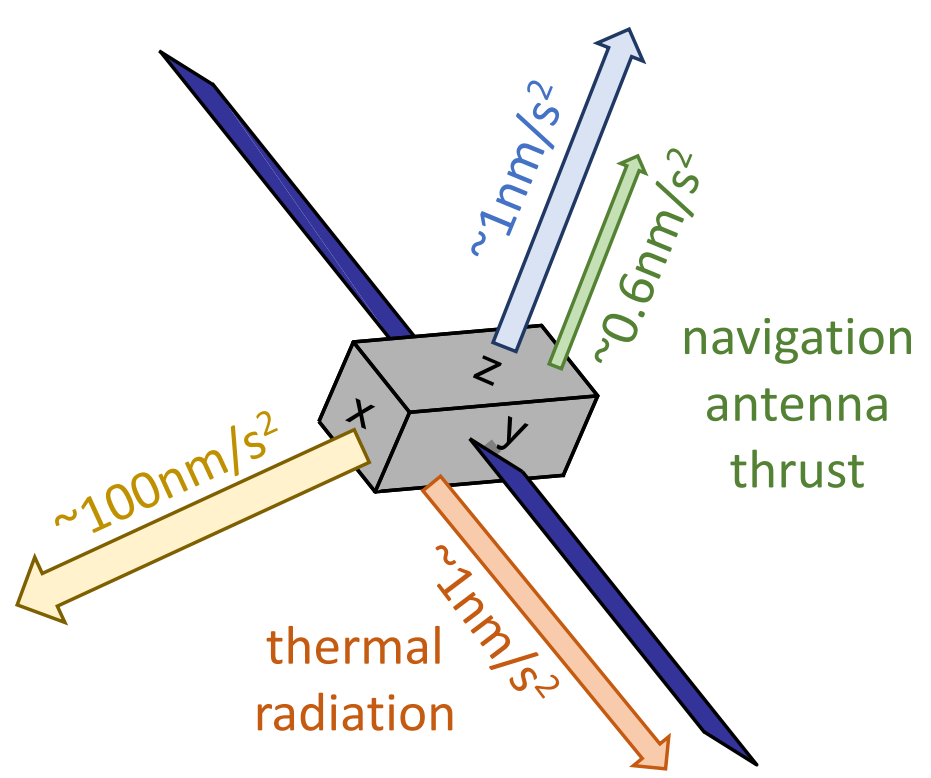

radiation

In this study, the propagation of uncertainties in a box-wing model (currently limited to the size of the plates)

into a GNSS solution is investigated. 


\section{Simulation setup - box-wing mismodelling}

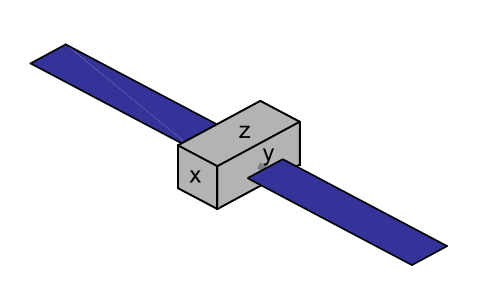

REFERENCE satellite box-wing model (used to generate REFERENCE solution)

case 1

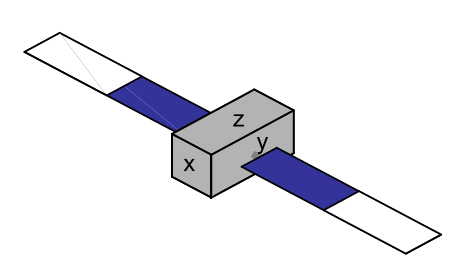

case 2

case 3

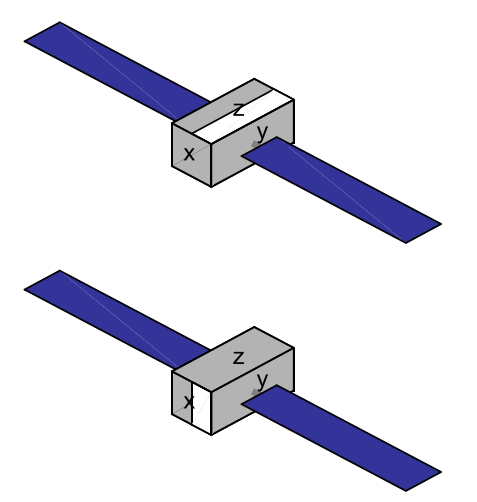

Note:

area of plane $\boldsymbol{-} \mathbf{z}$ and $\boldsymbol{+} \boldsymbol{z}$ (facing the Earth's surface) reduced by

$50 \%$

area of plane $-\boldsymbol{x}$ and $+\boldsymbol{x}$ reduced by $50 \%$

area of solar panels reduced by $50 \%$

Substantial box-wing mismodelling was applied to emphasise the effect in the solution. This can be scaled down to more reasonable values. 


\section{Results - residuals of initial orbit fit}

case 1
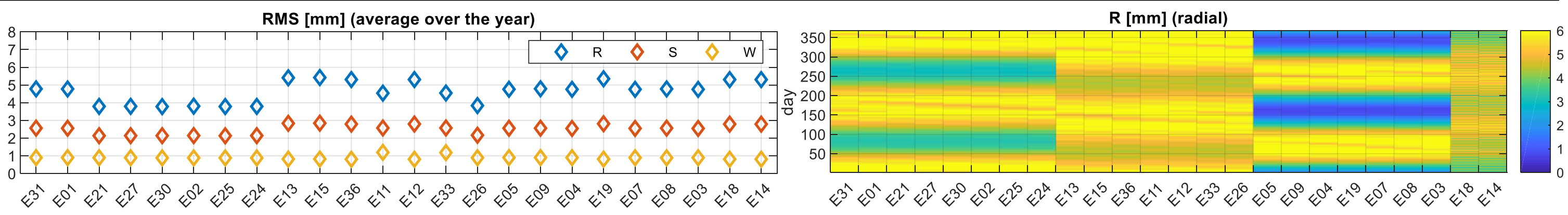

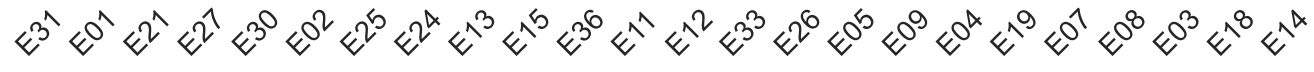

$S$ [mm] (along track)
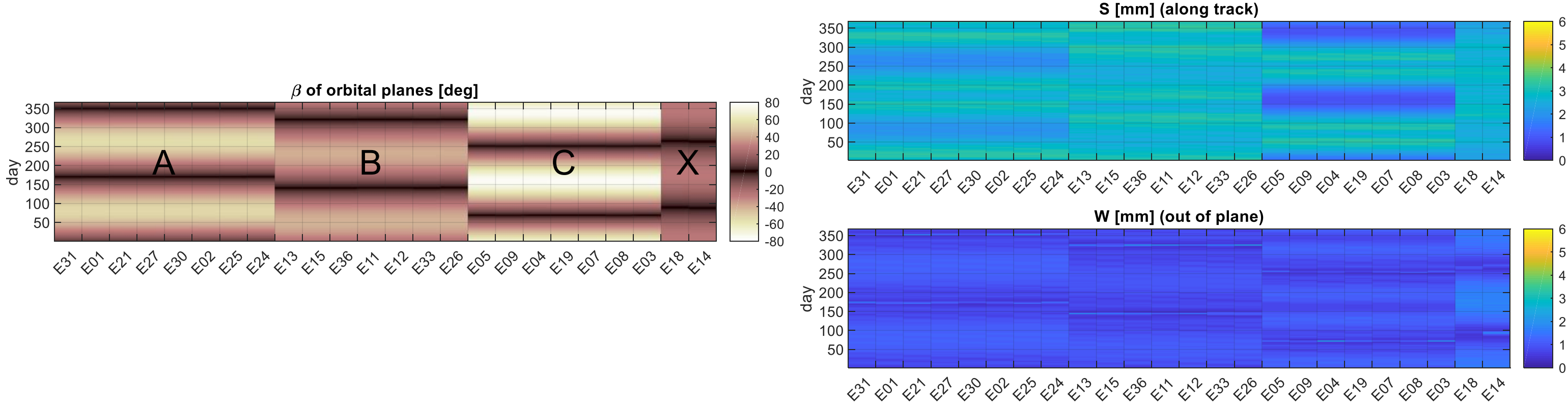

- ECOM2 cannot fully capture mismodelled NGP

- ECOM2 performs better for higher $\beta$ angles in case the NGP model assumes incorrect area of solar panel (in hypothetical case of $\beta=90^{\circ} \mathrm{NGP}$ mismodelling would be fully captured by $D_{0}$ coefficient)

Note: satellites are ordered according to the orbital plane and the slot 
Results - orbits: RMS error (ambiguities resolved)

epoch-wise clocks

L: $\mathbf{R}[\mathrm{mm}]$

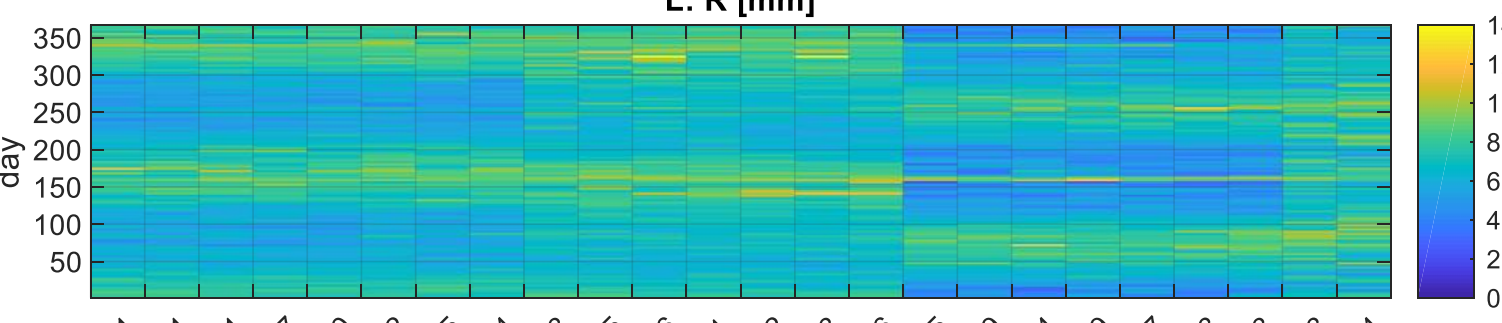

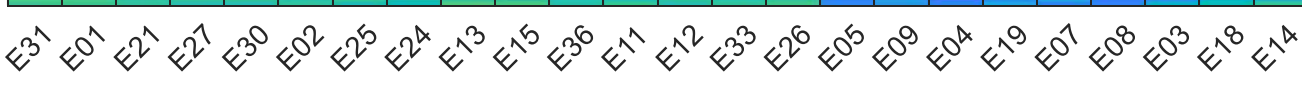

L: $\mathbf{S}$ [mm]

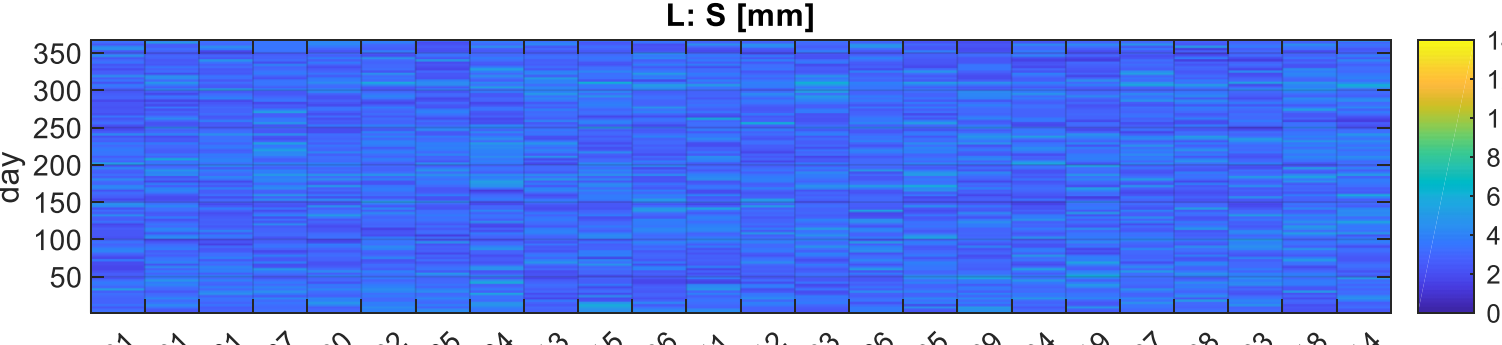

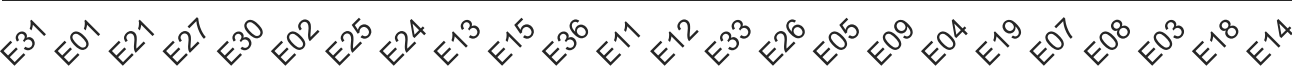

L: $\mathbf{W}$ [mm]

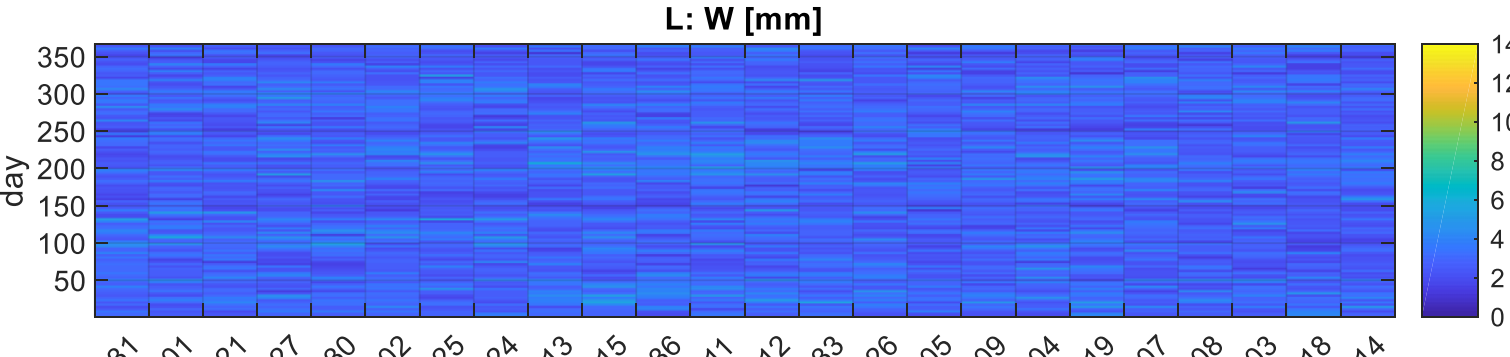

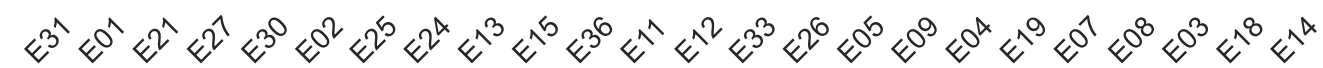

clock modelling applied

M: $\mathbf{R}[\mathrm{mm}]$

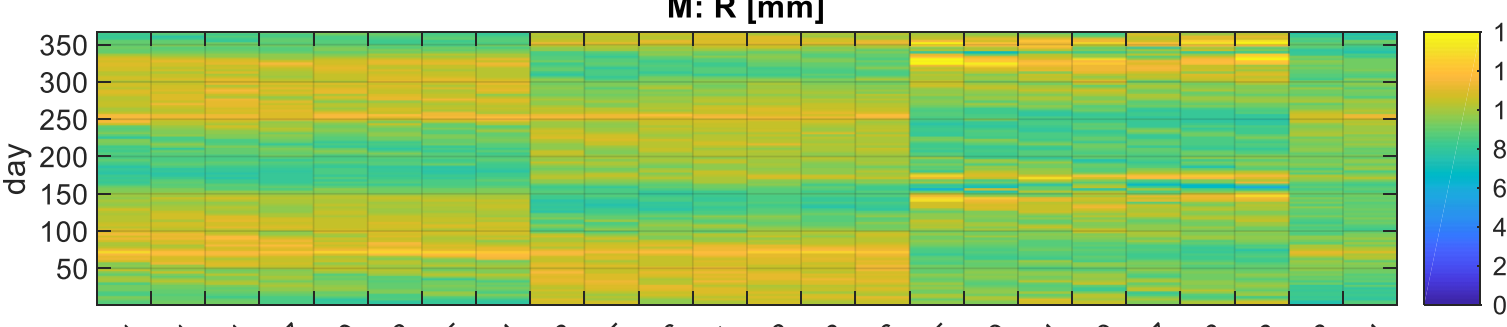

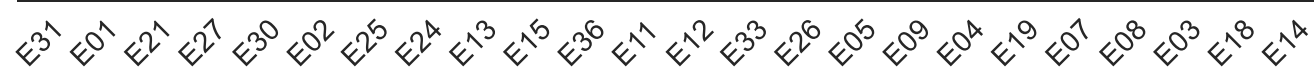

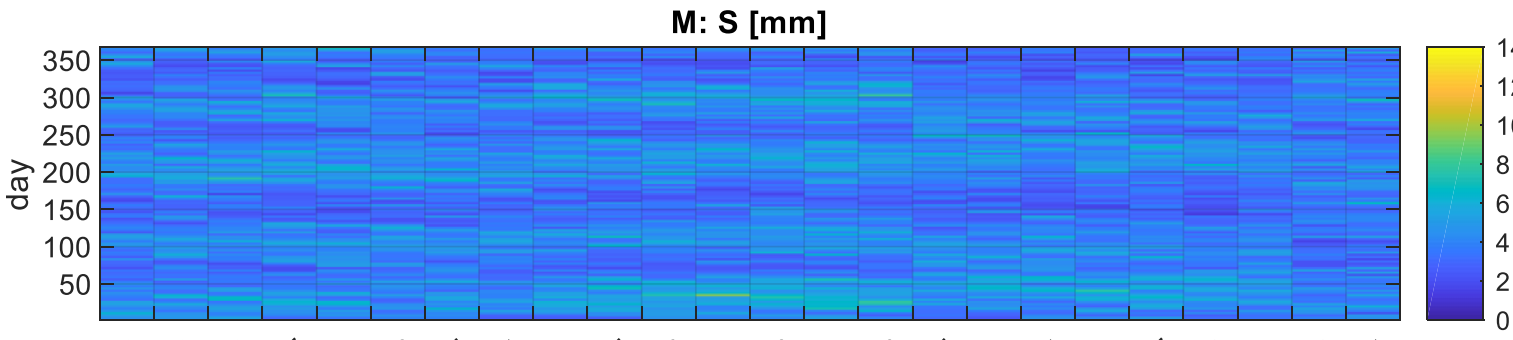

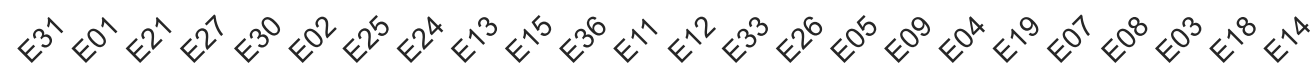

M: W [mm]

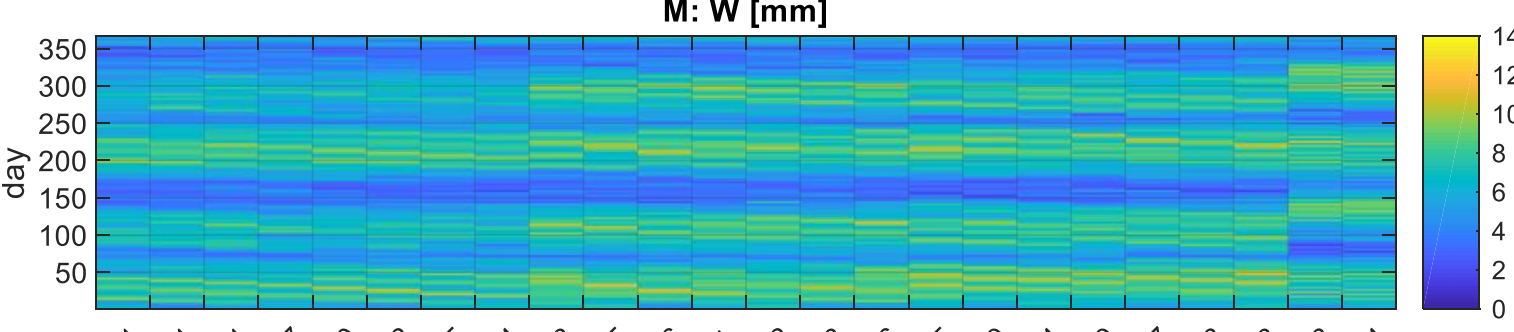

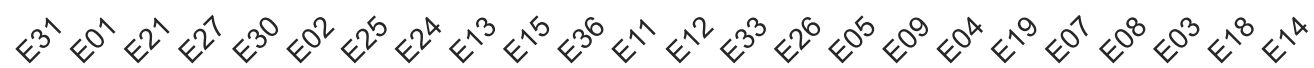

- clock modelling considerably reduces number of estimated parameters

(e.g. for one satellite the number of parameters drops from 288 epoch-wise (every $5 \mathrm{~min}$.) clock corrections to 2 values: offset and drift)

- orbit errors can be absorbed by epoch-wise estimated satellite clock corrections but not when the clocks are modelled 
Results - orbits: RMS error (float ambiguities)

case 1

epoch-wise clocks

L: $\mathbf{R}[\mathrm{mm}]$

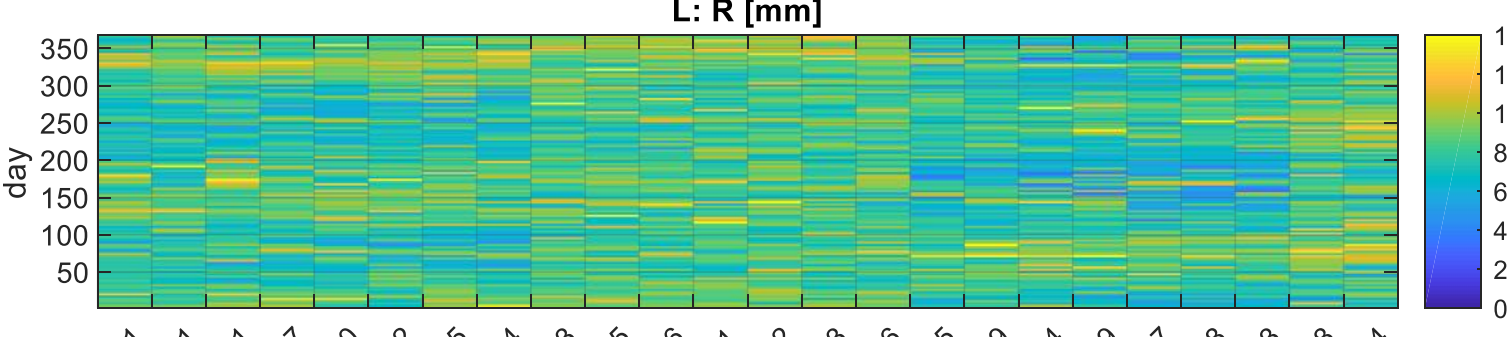

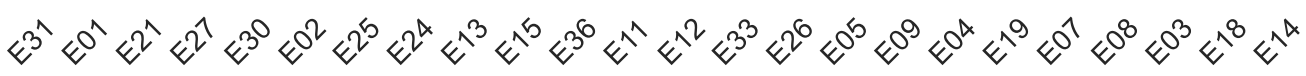

L: $\mathbf{S}[\mathrm{mm}]$

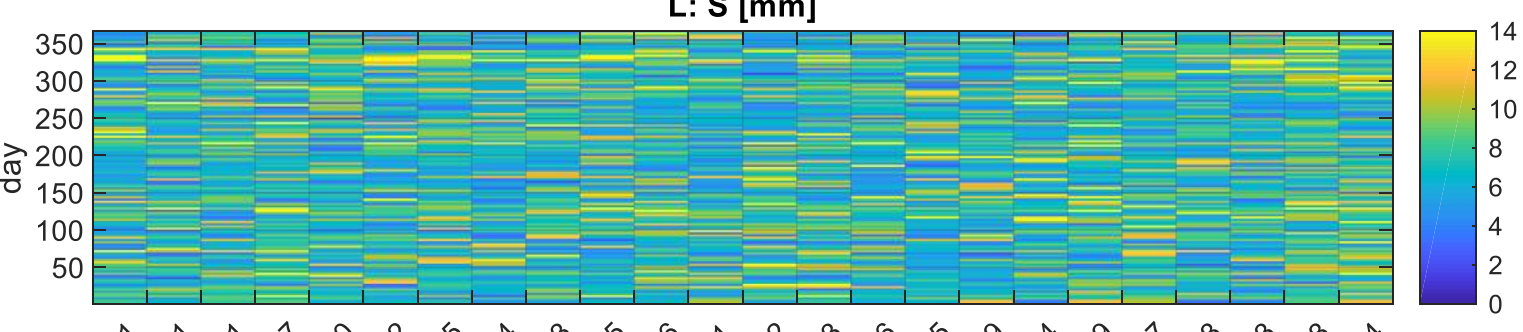

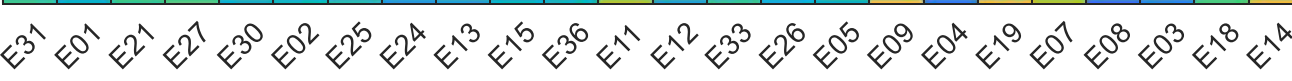

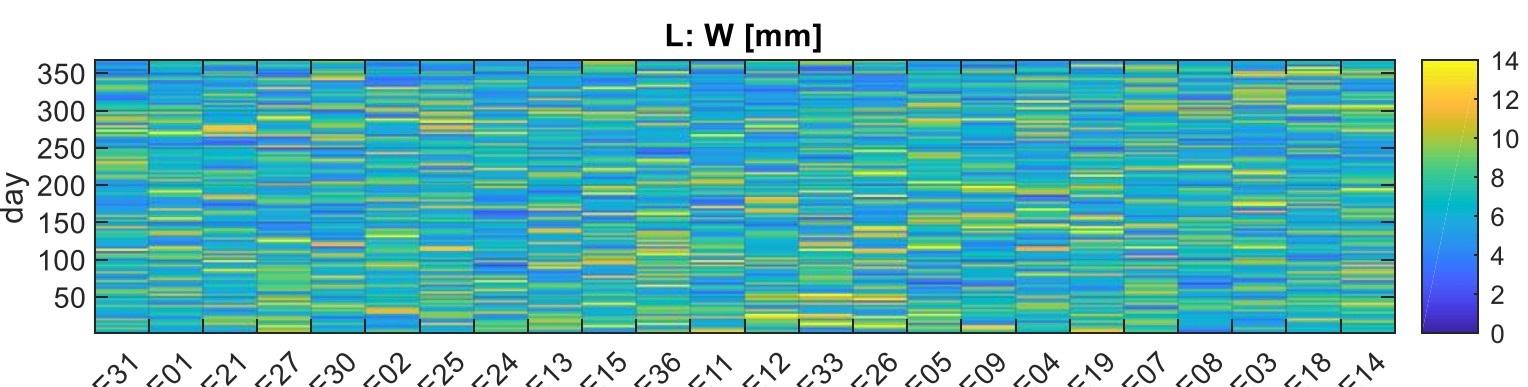

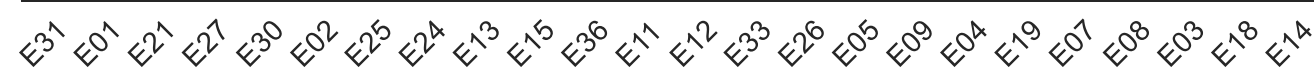

clock modelling applied

M: R [mm]

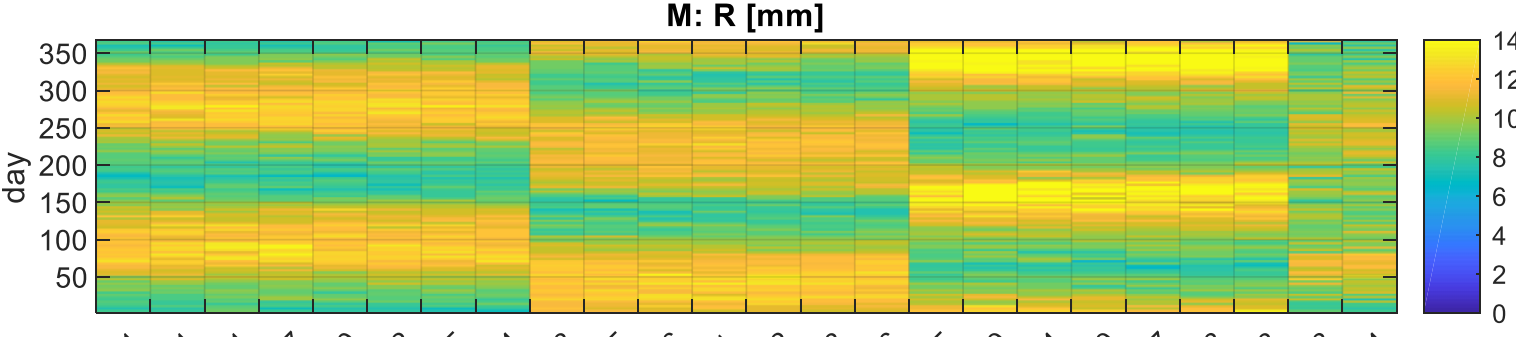

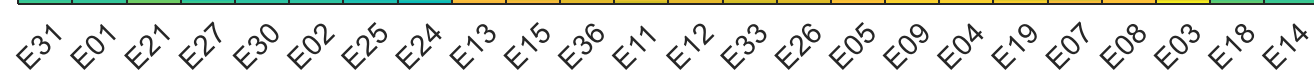

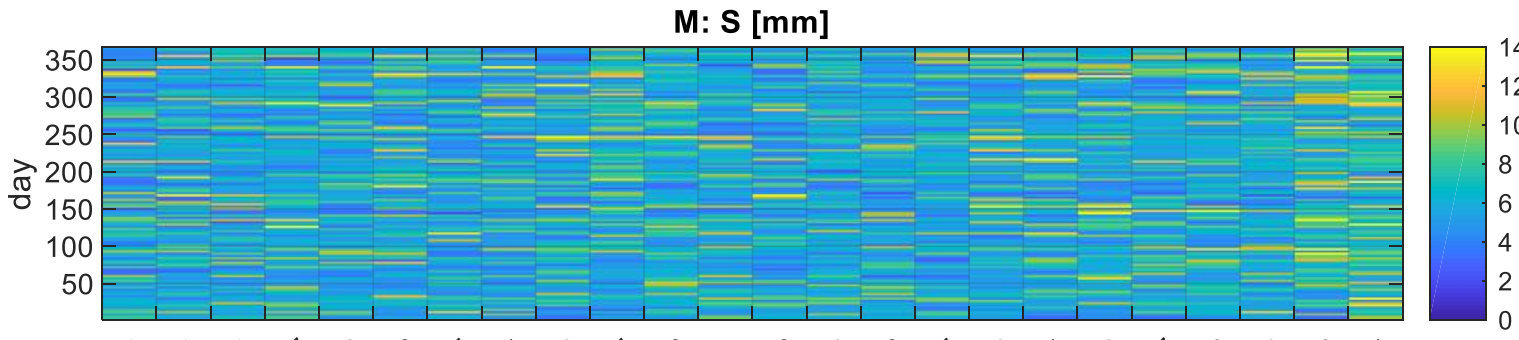

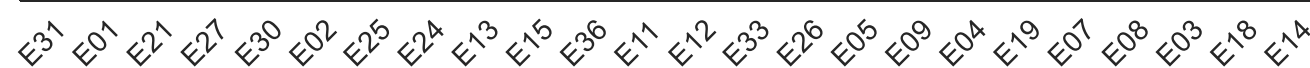

$\mathbf{M : ~ W}[\mathrm{mm}]$

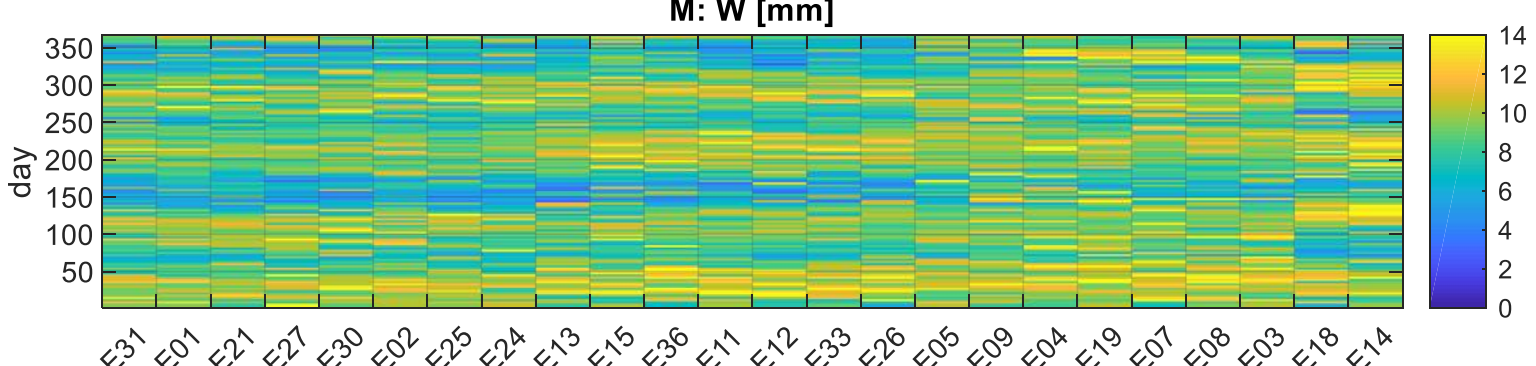

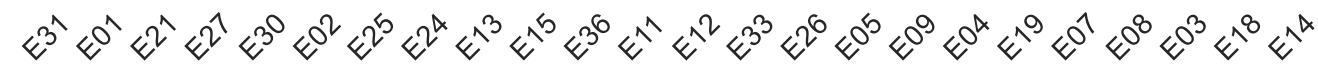

- also ambiguity parameters estimated to their real value can absorb a part of the orbit mismodelling (compare to previous slide) 


\section{RMS values averaged over the year}

epoch-wise clocks

L: RMS $[\mathrm{mm}]$

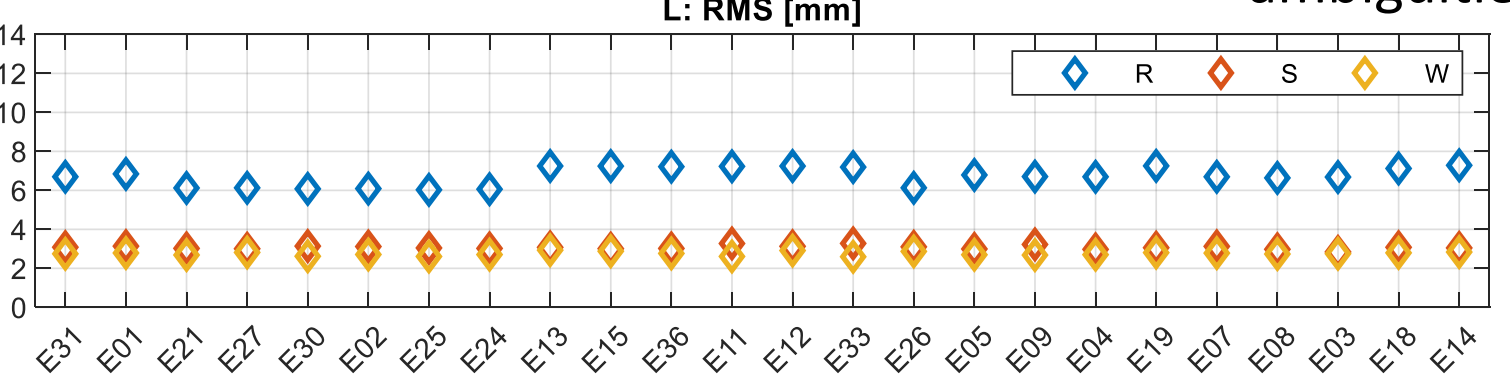

ambiguities resolved clock modelling applied

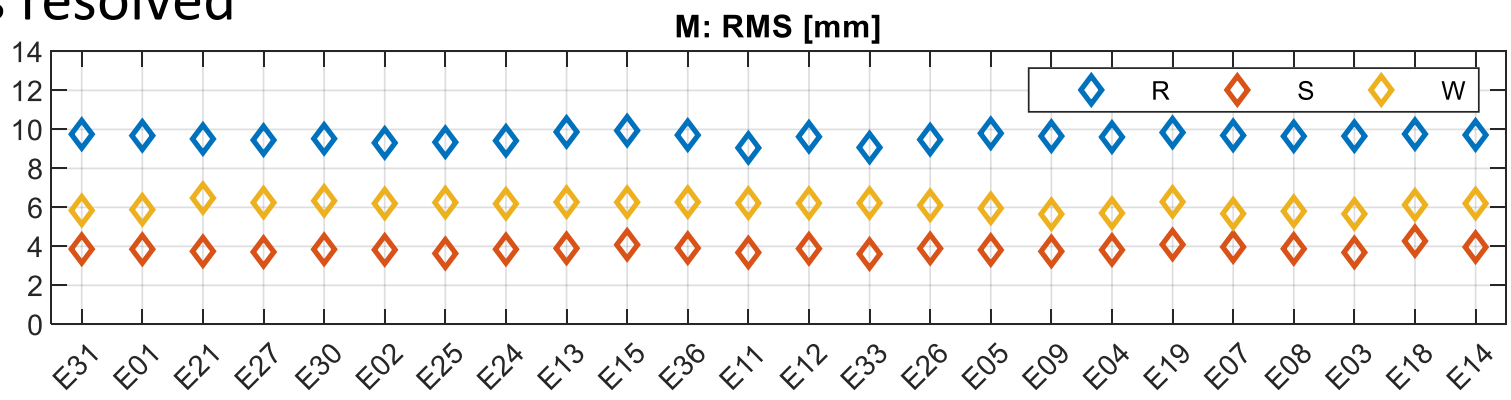

float ambiguities

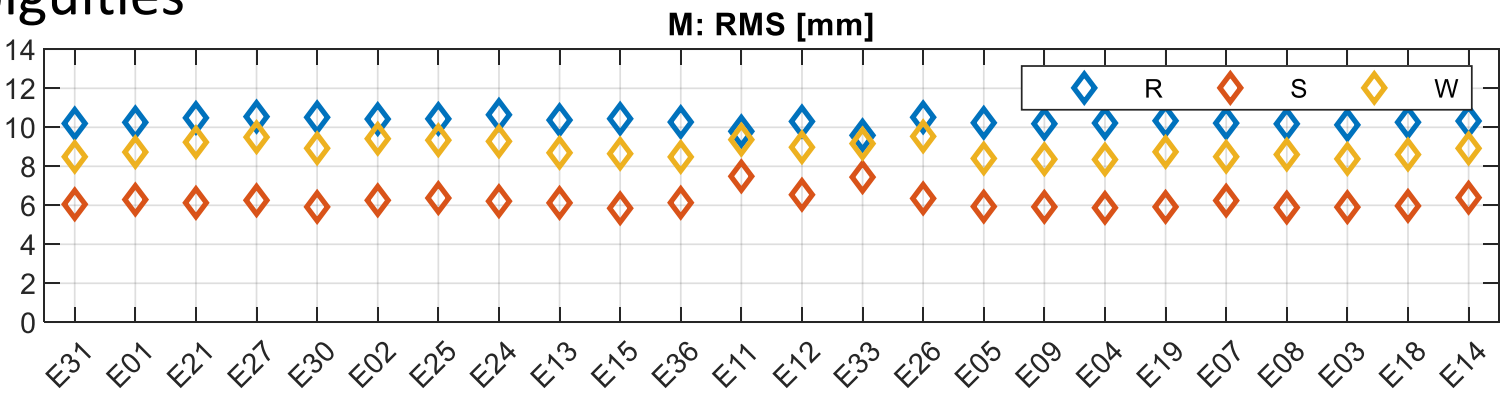

- estimation strategy (including applied models, constraints, ...) has a clear impact on the results

- the more degrees of freedom is provided by the parametrization (epoch-wise satellite clock corrections, estimated ambiguity parameters freely estimated as real numbers), the more from the (mainly radial) orbit errors is absorbed. 
Results - ECOM2 constant terms (ambiguities resolved)

epoch-wise clocks

L: $D_{0}\left[\mathrm{~nm} / \mathrm{s}^{2}\right]$

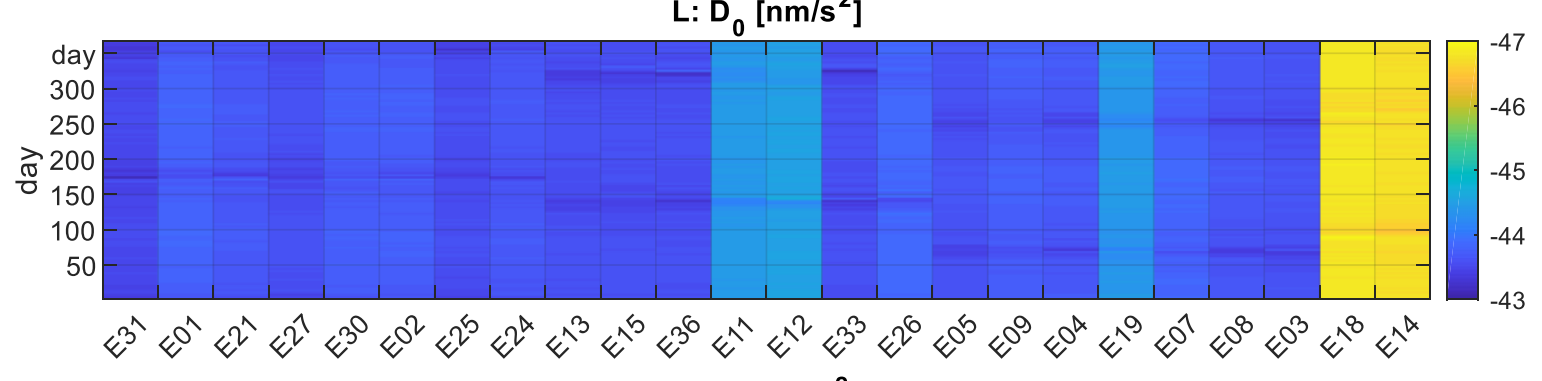

L: $Y_{0}\left[\mathrm{~nm} / \mathrm{s}^{2}\right]$

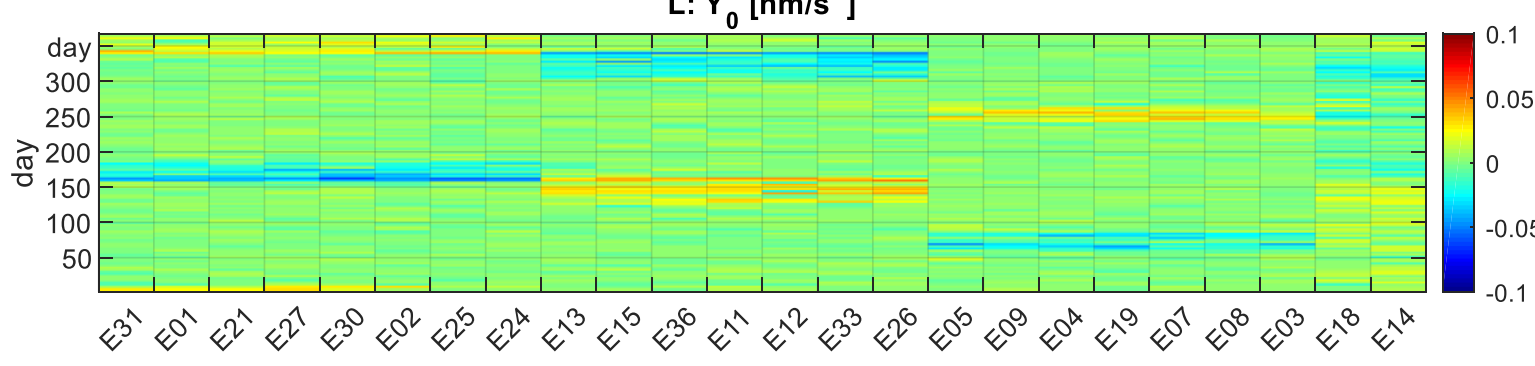

L: $B_{0}\left[\mathrm{~nm} / \mathrm{s}^{2}\right]$

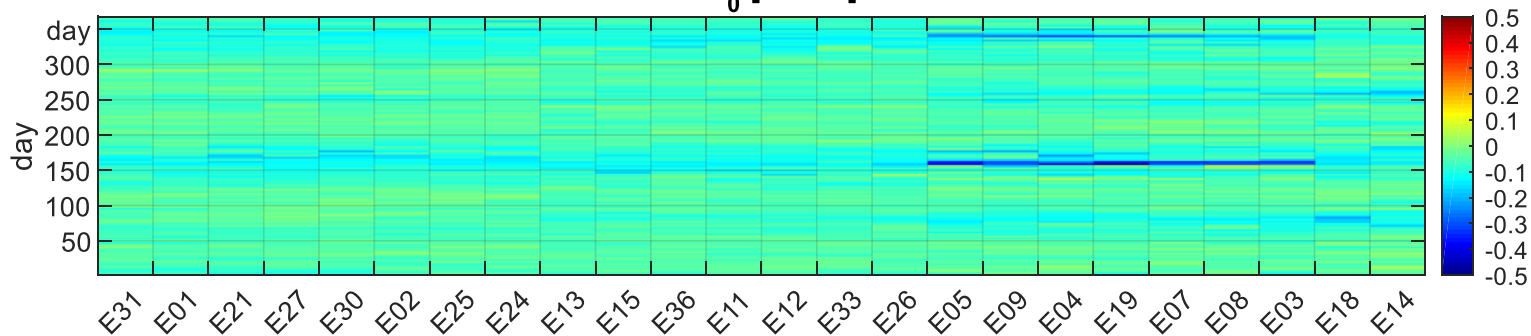

clock modelling applied

$M: D_{0}\left[\mathrm{~nm} / \mathrm{s}^{2}\right]$

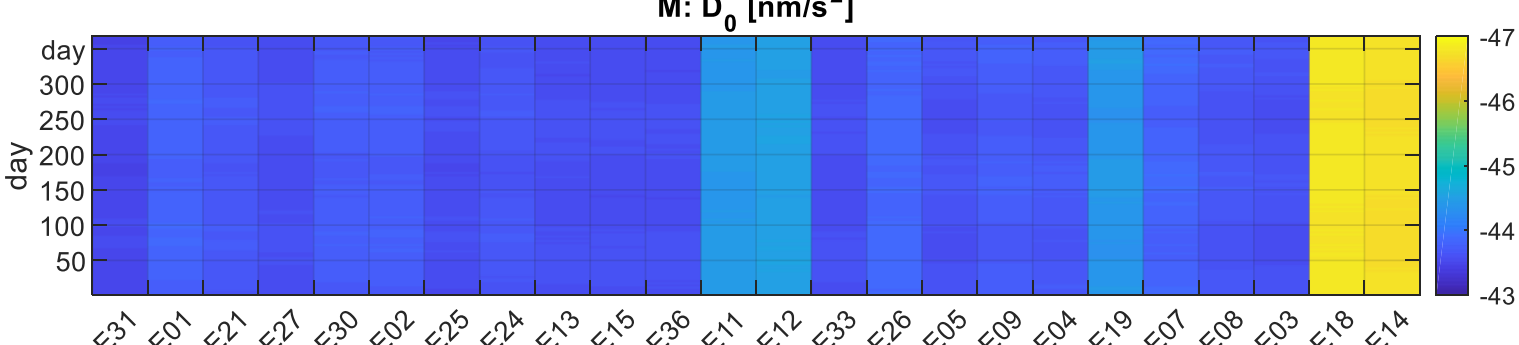

$M: Y_{0}\left[\mathrm{~nm} / \mathrm{s}^{2}\right]$

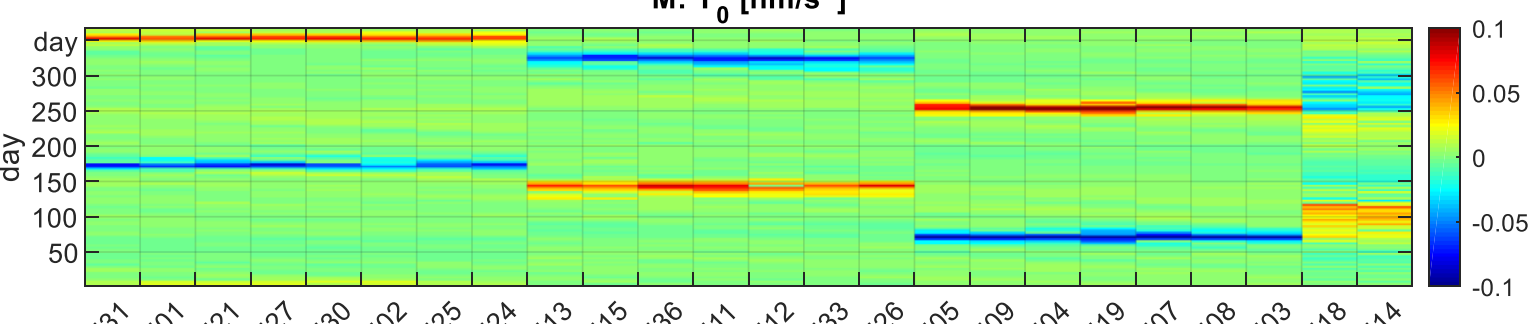

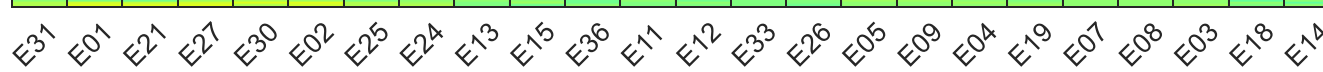

$M: B_{0}\left[\mathrm{~nm} / \mathrm{s}^{2}\right]$

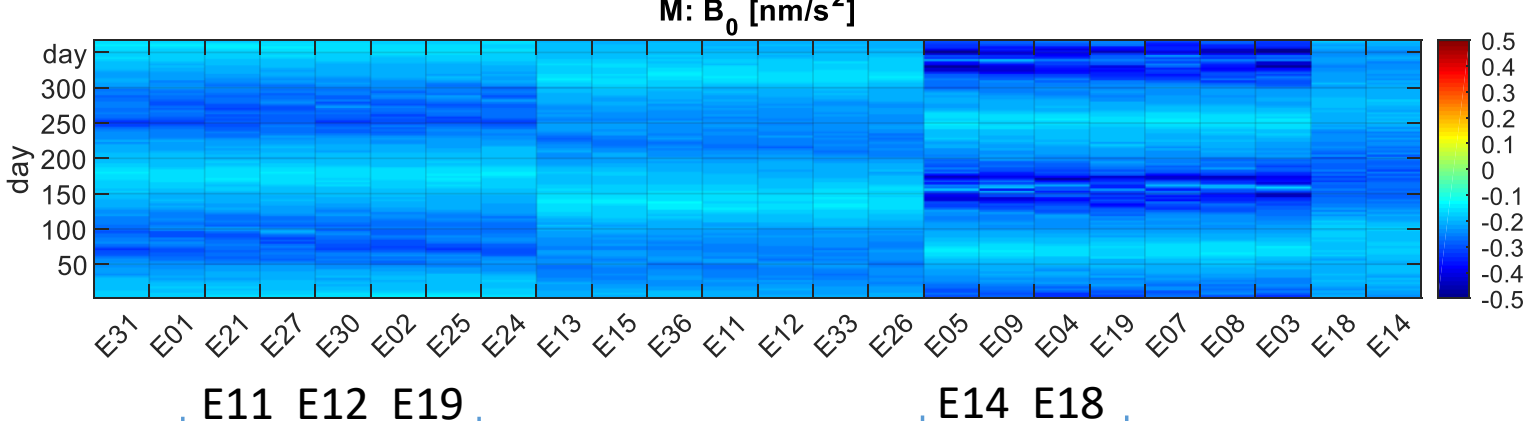

- $D_{0}$ captures the main part of the mismodelled NGP (different values for IOV, FOC and satellites on elliptical orbits can be observed)

- $Y_{0}$ partially captures NGP mismodelling due to the modified yaw steering law that is applied for small $\beta$ angles

- $\mathrm{B}_{0}$ coefficient is more pronounced (especially for higher $\beta$ angles) if clock modelling is applied 


\section{Results - ECOM2 periodic terms (ambiguities resolved)}

epoch-wise clocks

L: $\mathrm{D}_{\mathrm{C} 2}\left[\mathrm{~nm} / \mathrm{s}^{2}\right]$

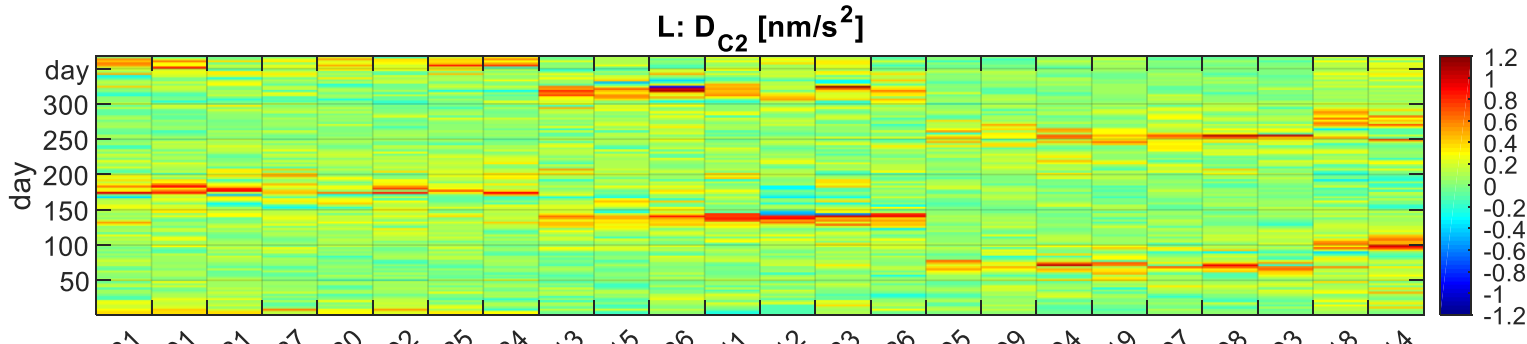

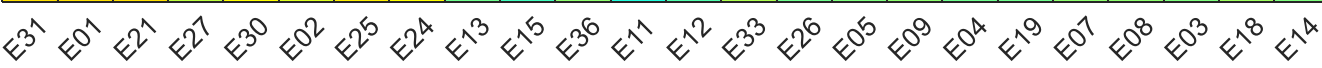
L: $\mathrm{D}_{\mathrm{s} 2}\left[\mathrm{~nm} / \mathrm{s}^{2}\right]$

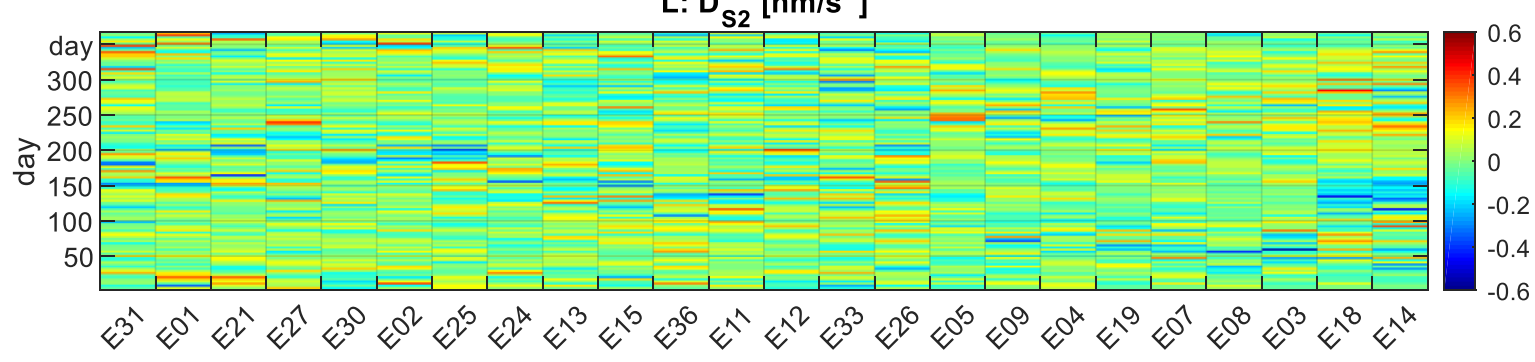

L: $B_{\mathrm{C} 1}\left[\mathrm{~nm} / \mathrm{s}^{2}\right]$

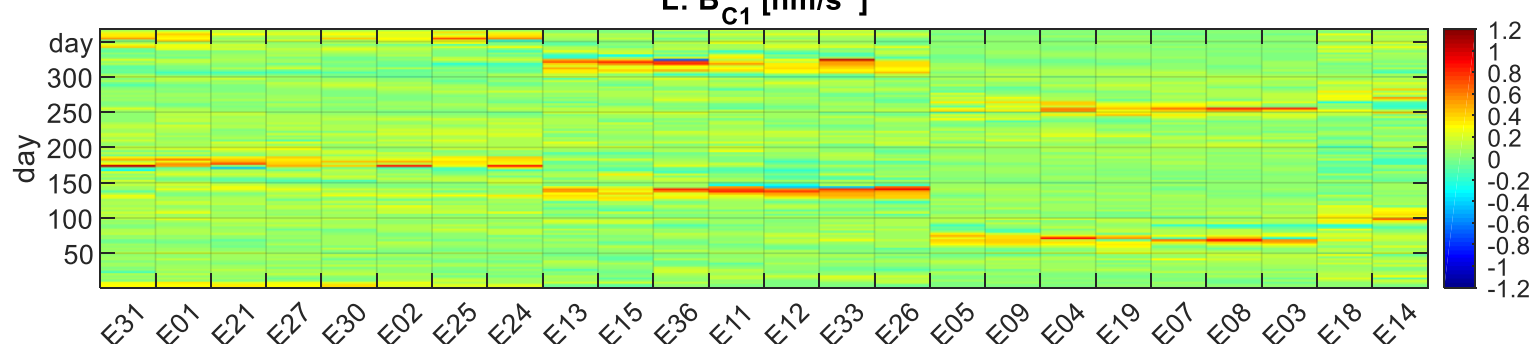

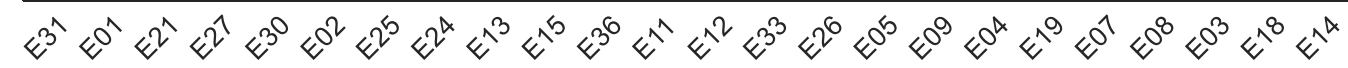

clock modelling applied

$\mathrm{M}: \mathrm{D}_{\mathrm{C} 2}\left[\mathrm{~nm} / \mathrm{s}^{2}\right]$

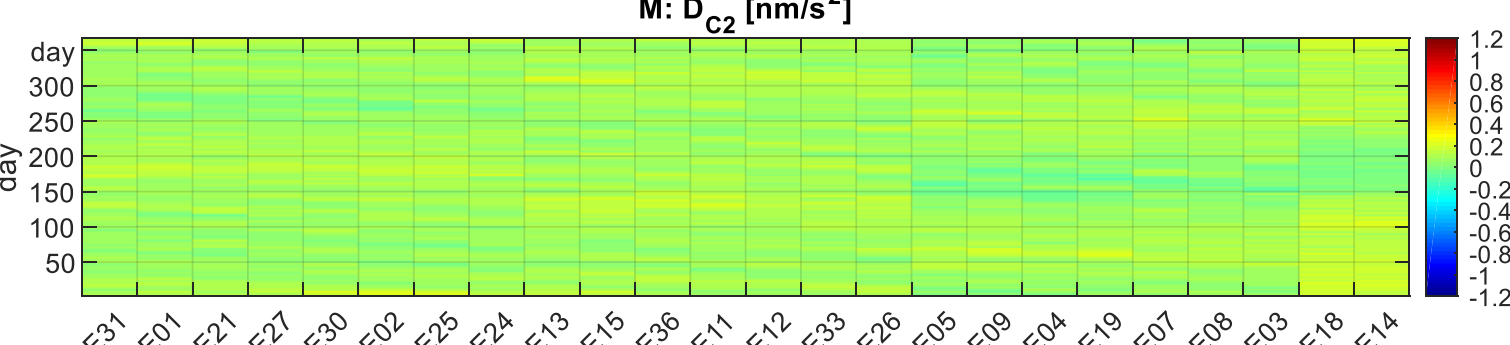

$\mathrm{M}: \mathrm{D}_{\mathrm{S} 2}\left[\mathrm{~nm} / \mathrm{s}^{2}\right]$

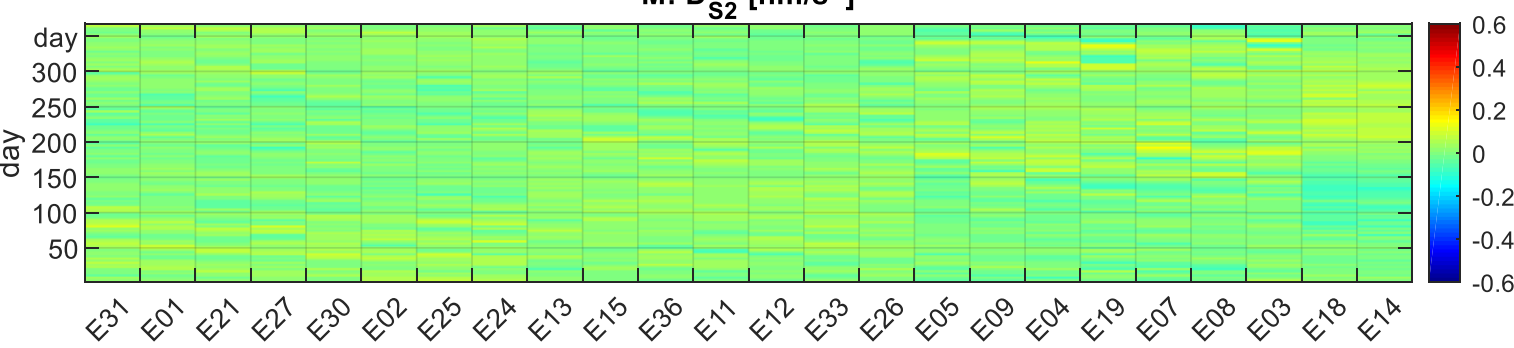

$M: B_{C 1}\left[\mathrm{~nm} / \mathrm{s}^{2}\right]$

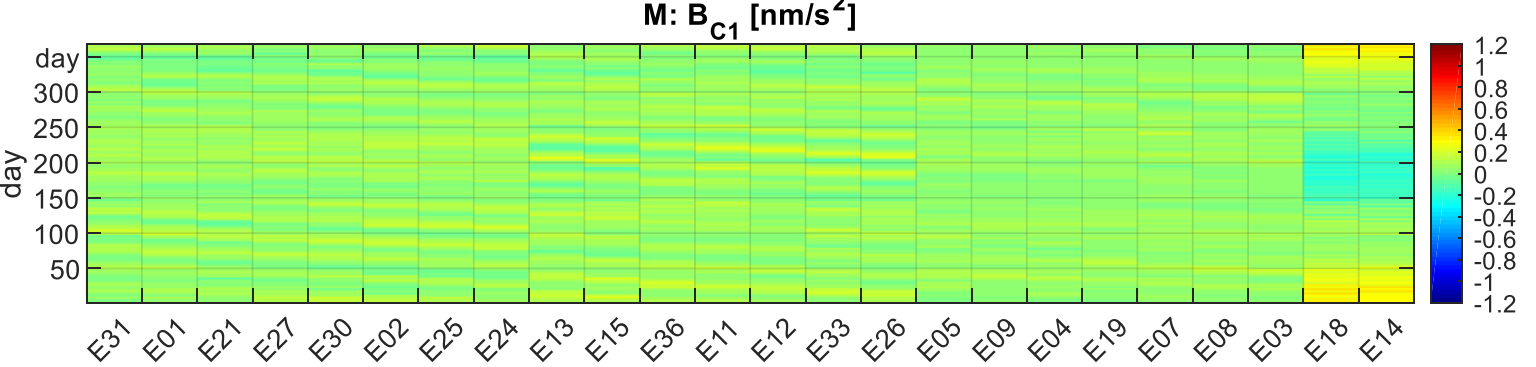

- scatter of ECOM2 parameters is considerably reduced by application of satellite clock modelling 


\section{Results - station coordinates (ambiguities resolved)}

epoch-wise clocks

$\mathrm{L}: \mathrm{U}$ [mm] equator

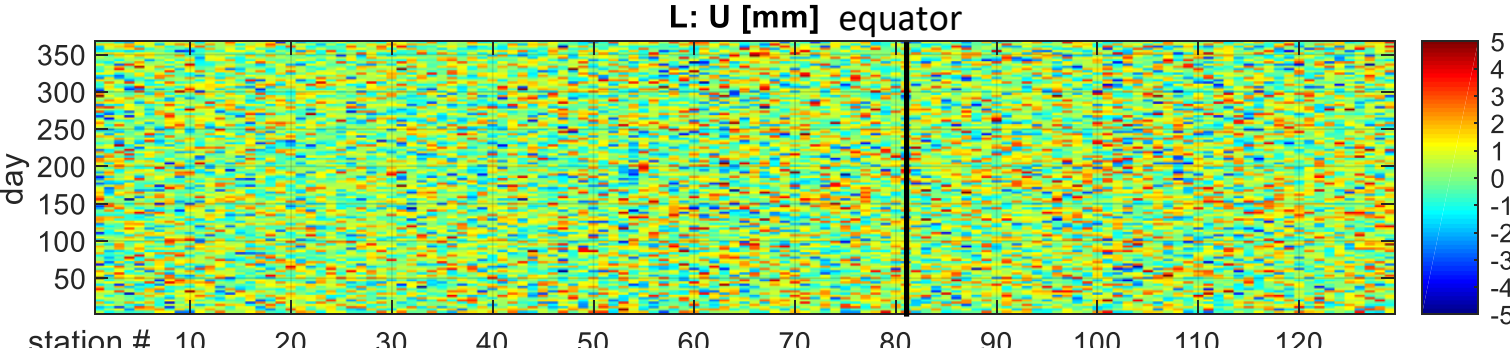

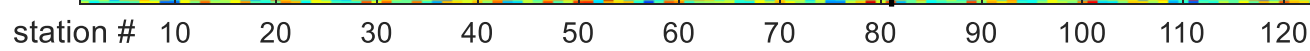

L: $\mathbf{N}[\mathrm{mm}]$

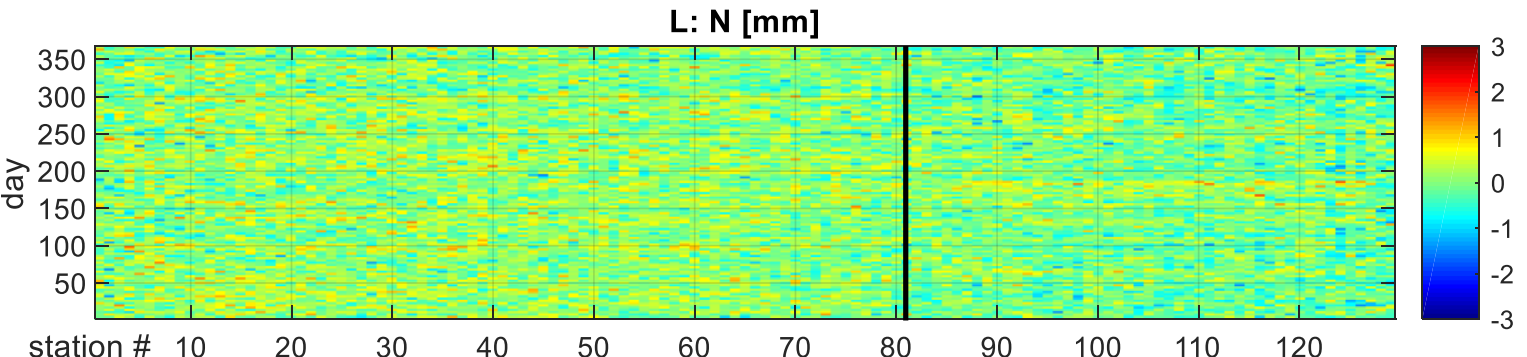

L: $E$ [mm]

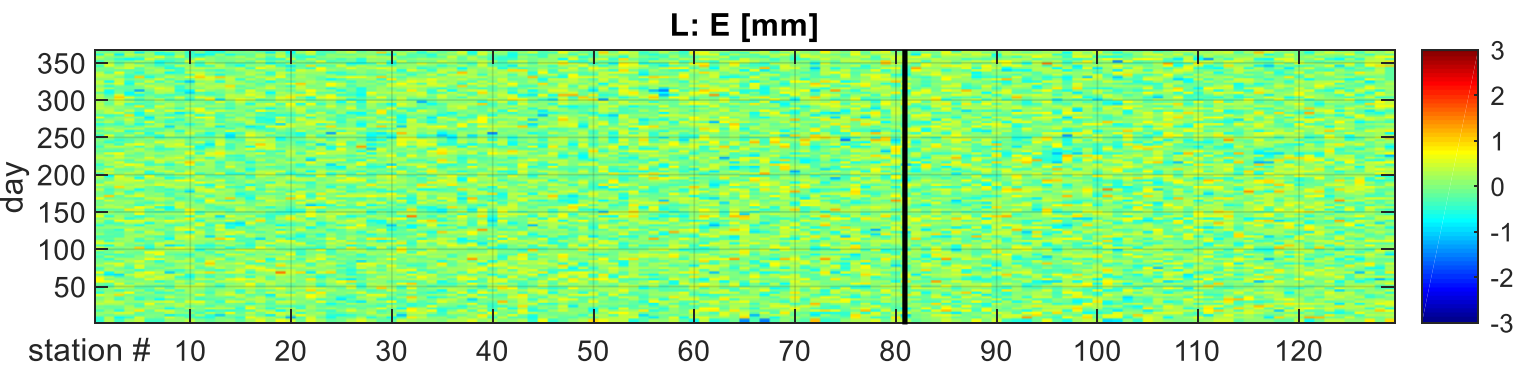

clock modelling applied

M: U [mm] equator

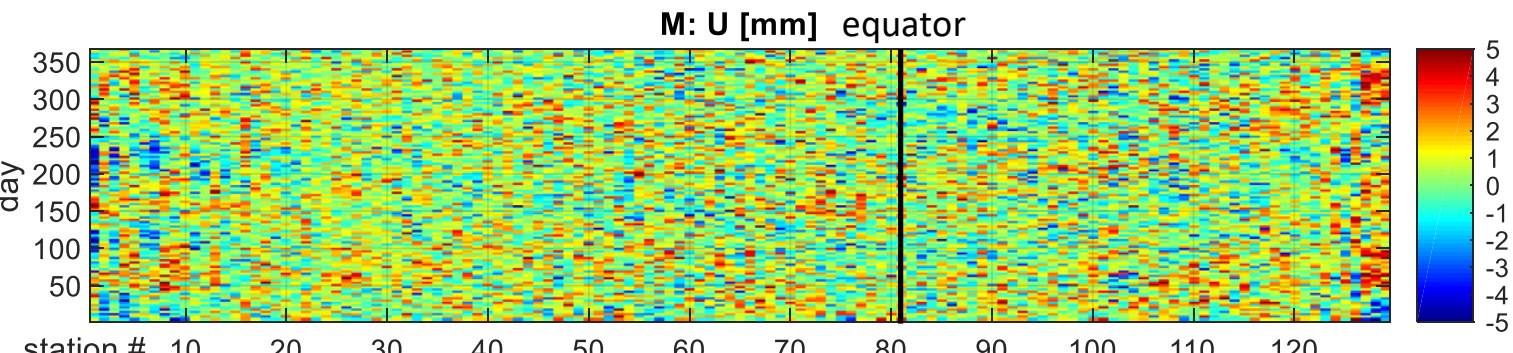

$\mathrm{M}: \mathbf{N}[\mathrm{mm}]$

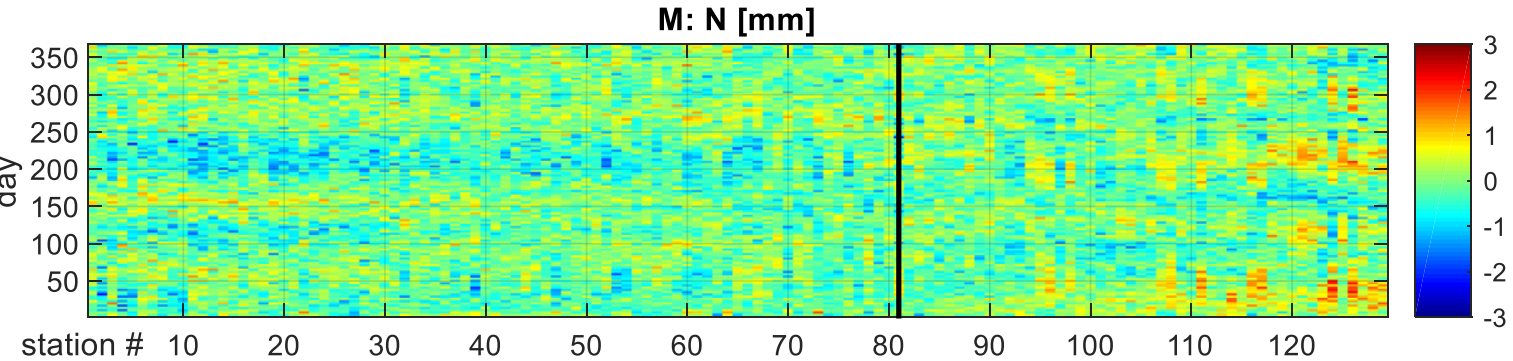

M: $E[\mathrm{~mm}]$

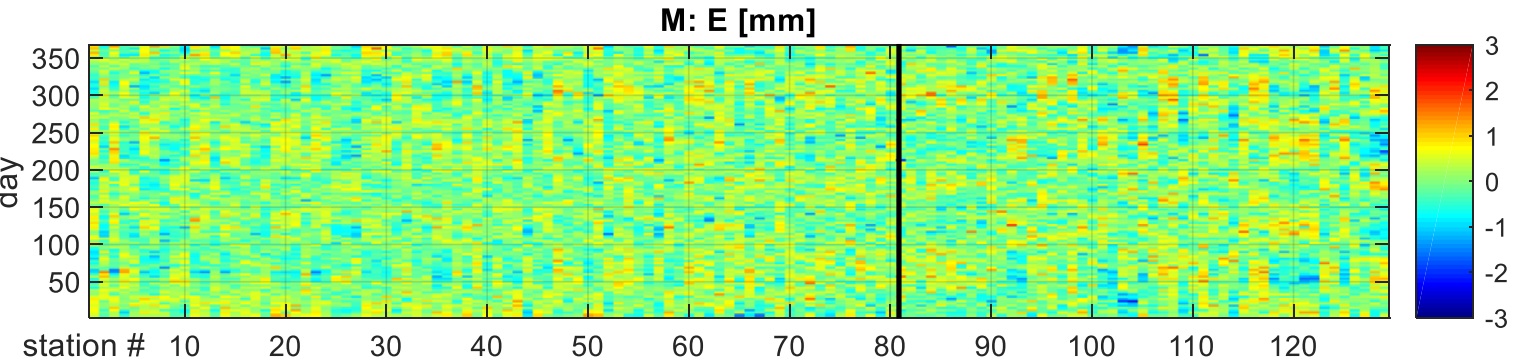

- stations coordinates are more affected by NGP mismodelling if satellite clock modelling is applied

- effect is more pronounced for stations with higher latitude

- results for 'up' component reflect the radial orbit errors

Note: stations are ordered according to the latitude (north $\Rightarrow$ south) 


\section{Results - station coordinates (float ambiguities)}

epoch-wise clocks

L: $U$ [mm]

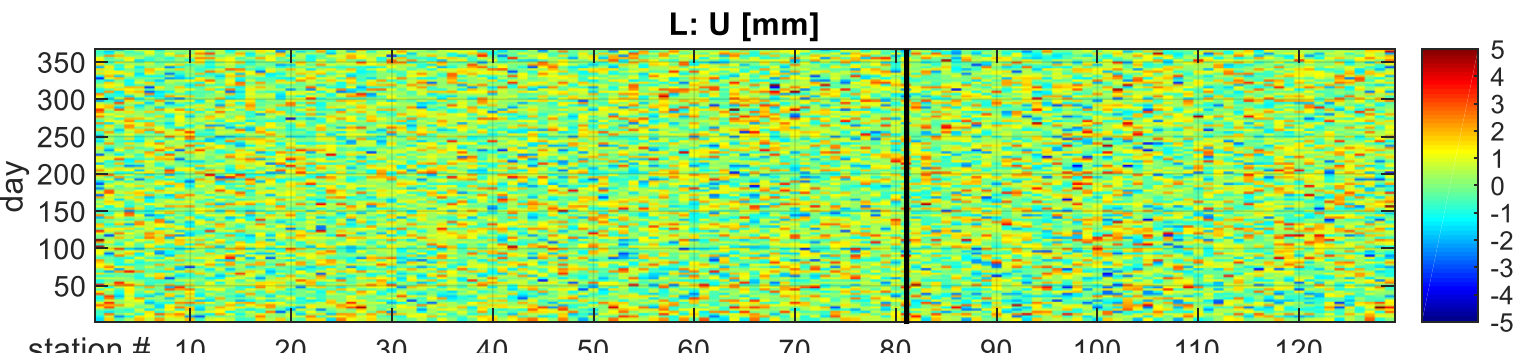

L: $\mathbf{N}[\mathrm{mm}]$

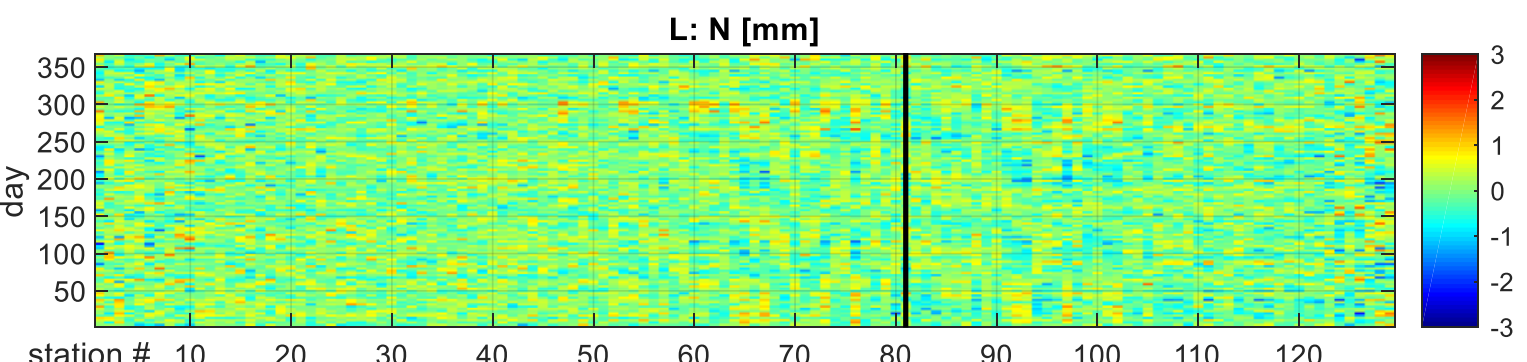

L: $E$ [mm]

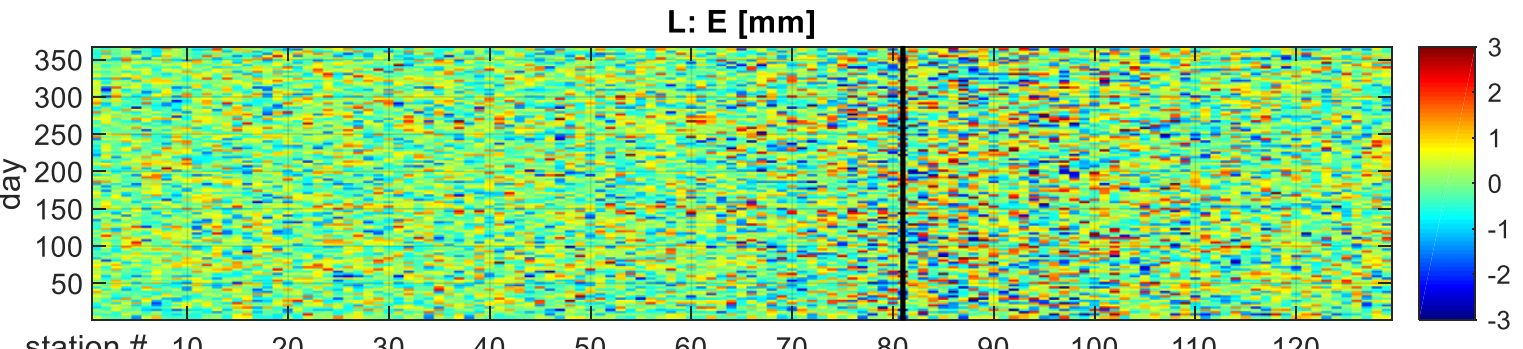

clock modelling applied

$\mathrm{M:} \mathrm{U}[\mathrm{mm}]$

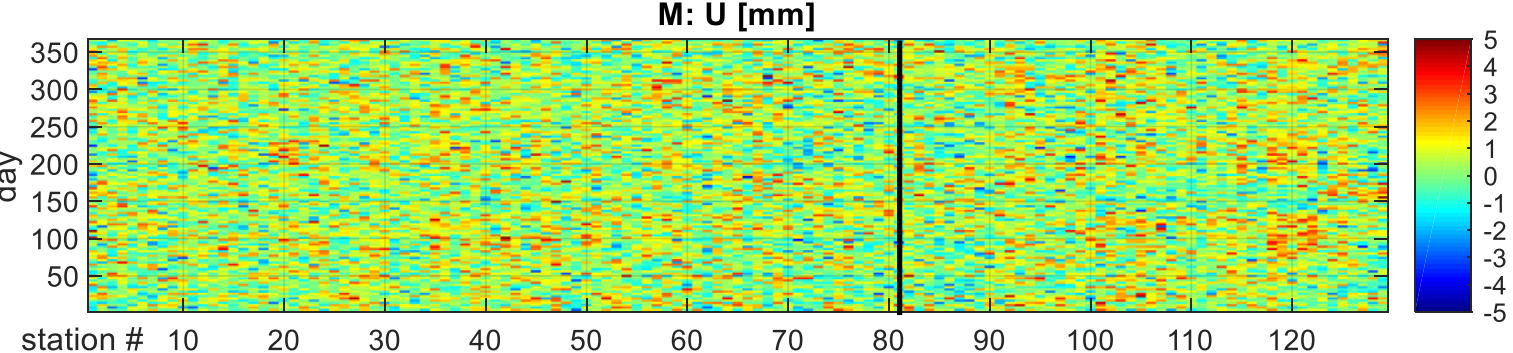

M: N [mm]

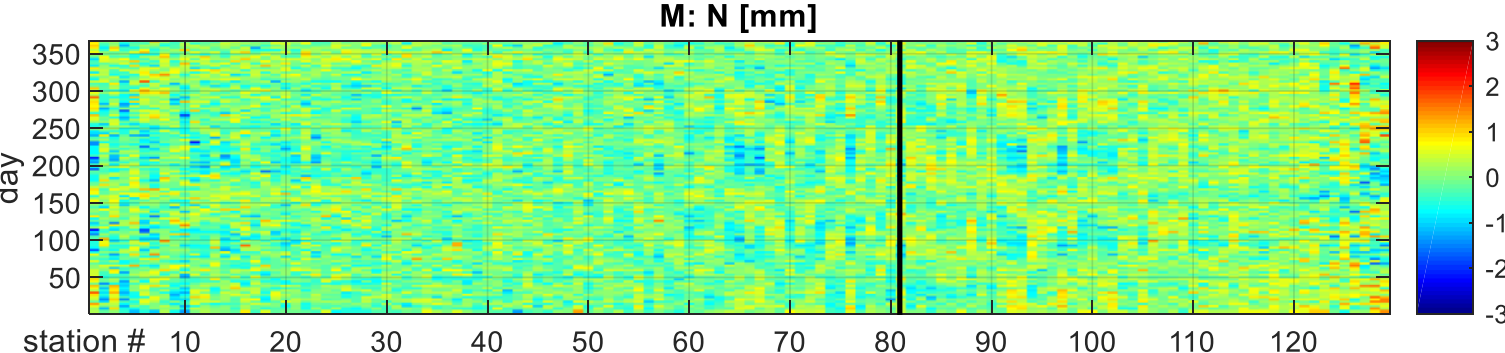

M: $\mathrm{E}[\mathrm{mm}]$

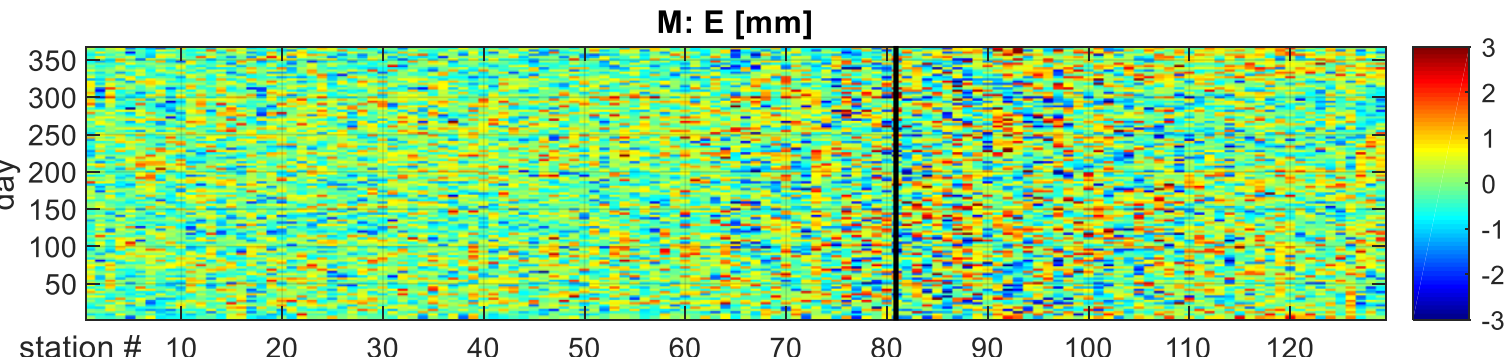

- 'up' component is less noisy if float ambiguities remain in the system

- asymmetric result for 'east' component reflects the uneven distribution of stations (more on the northern hemisphere)

- stations closer to equator are more affected by NGP mismodelling 
Results - satellite clocks (ambiguities resolved) case 1
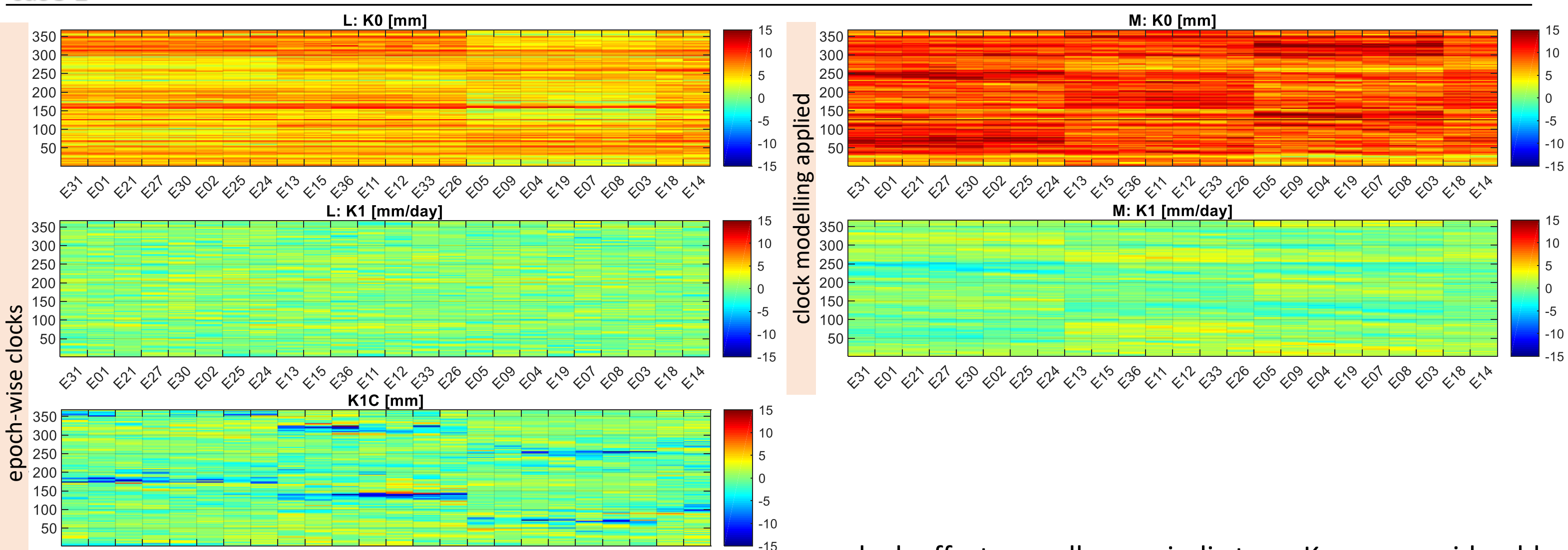

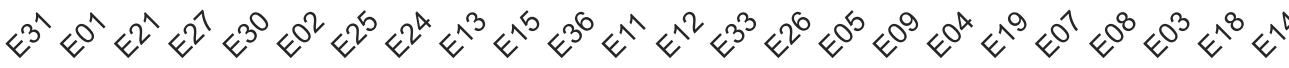

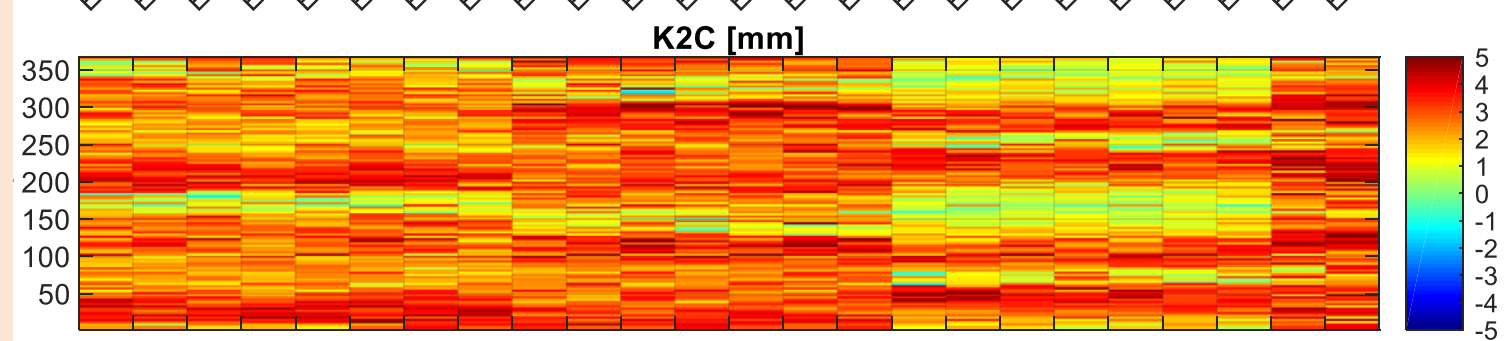

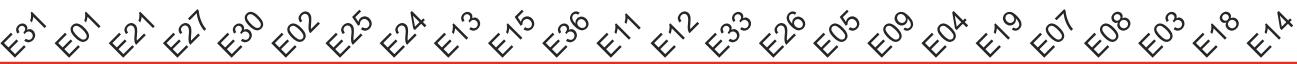

- clock offset as well as periodic term $\mathrm{K}_{2 \mathrm{C}}$ are considerably affected by mismodelled NGP

L: $\Delta t_{s a t}(t, u)=K_{o}+K_{1} t+K_{1 C} \cos (u)+K_{1 S} \sin (u)+$

$+K_{2 C} \cos (2 u)+K_{2 S} \sin (2 u)+K_{4 C} \cos (4 u)+K_{4 S} \sin (4 u)$

M: $\Delta t_{s a t}(t, u)=K_{o}+K_{1} t$ 


\section{Results - orbits RMS error (ambiguities resolved)}

\section{case 2}
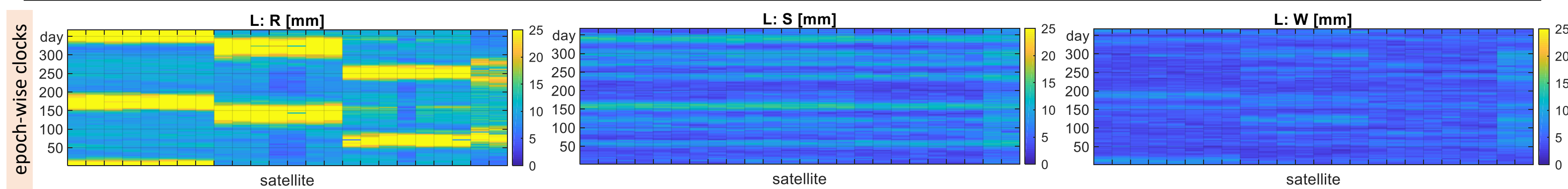

M: R [mm]

M: S [mm]
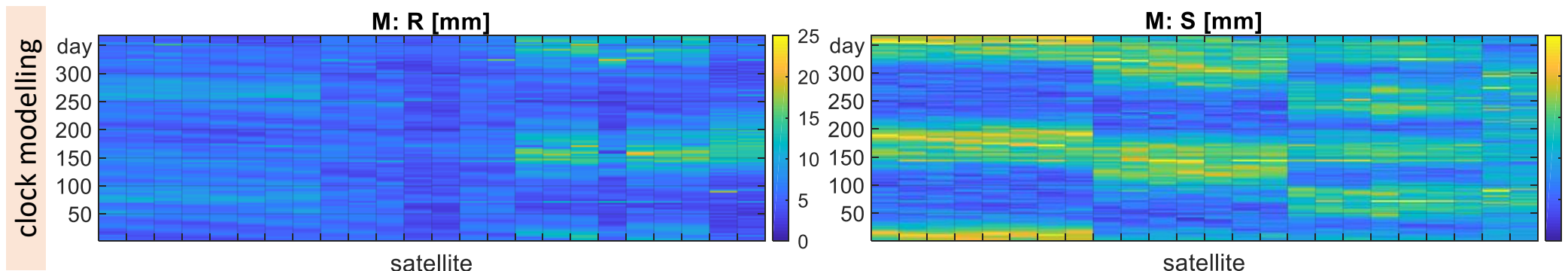

satellite

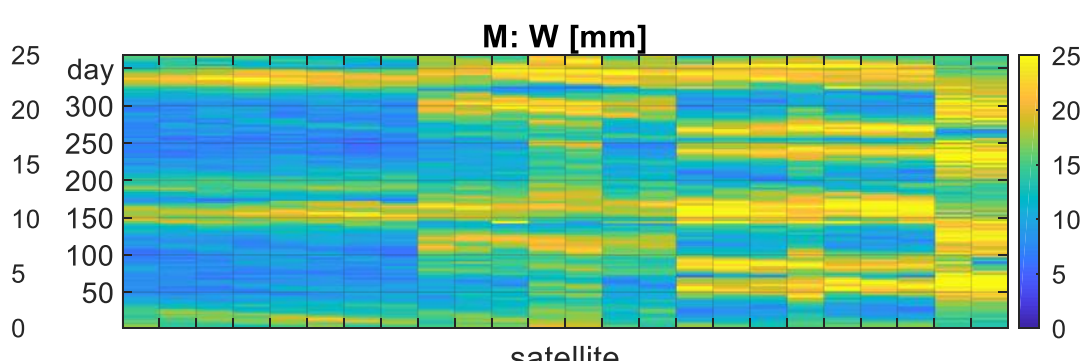

satellite

epoch-wise clocks

L: RMS [mm]

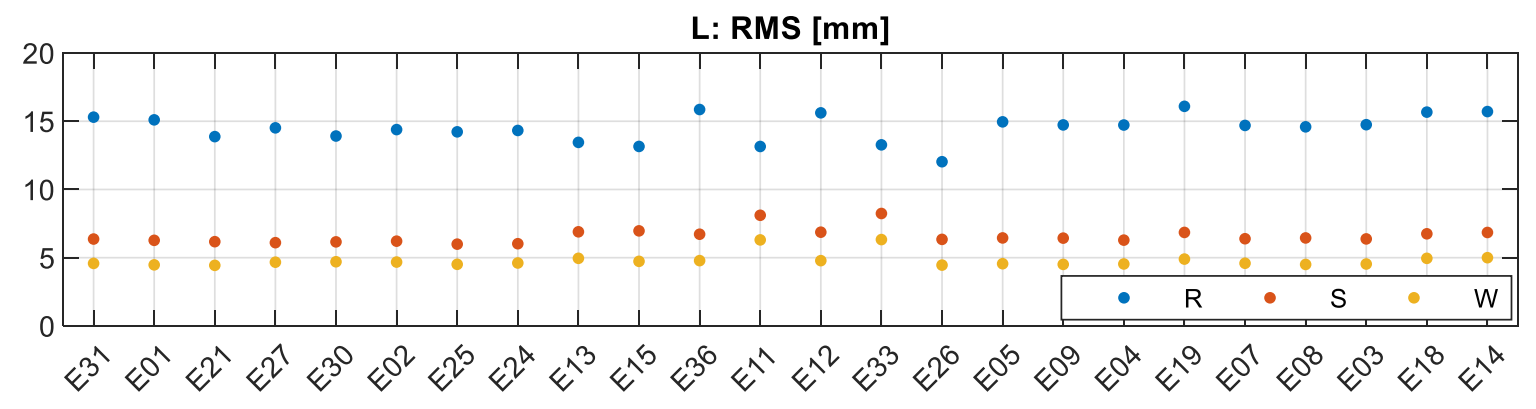

clock modelling applied

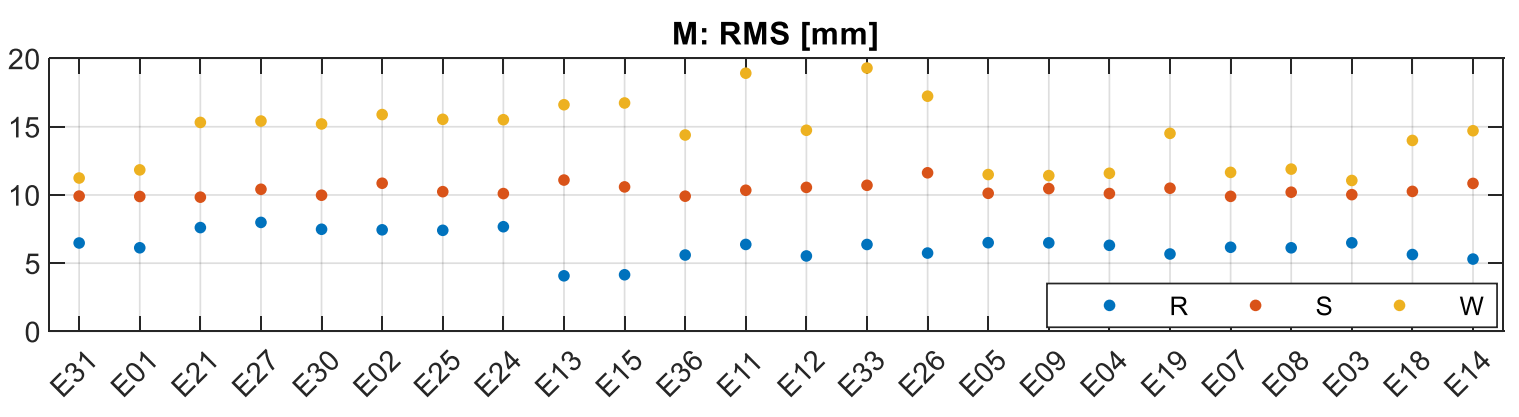

- different origins of NGP mismodelling lead to different pattern observed in orbit residuals 


\section{Results - orbits RMS error (ambiguities resolved)}

\section{case 3}
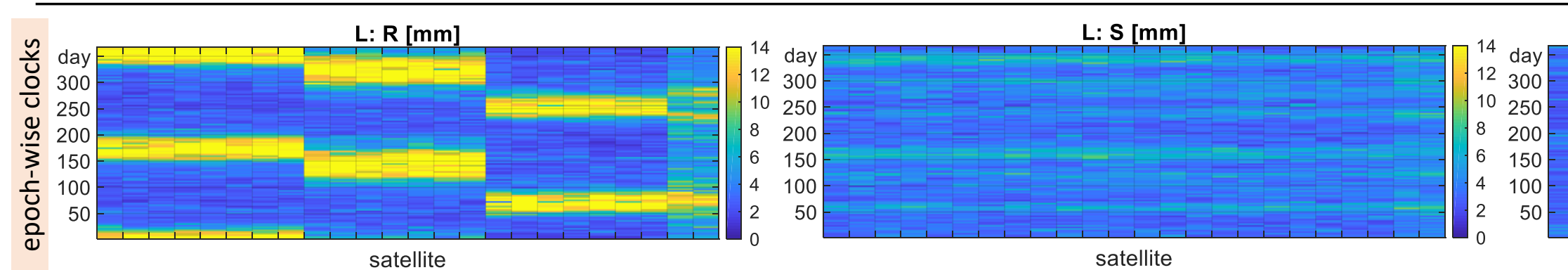

L: $\mathbf{W}[\mathrm{mm}]$

M: S [mm]
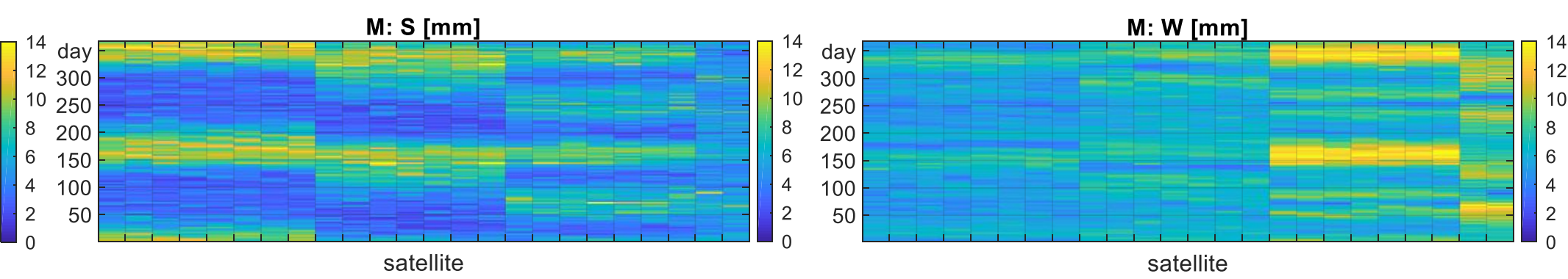

satellite

satellite

satellite

epoch-wise clocks

L: RMS [mm]

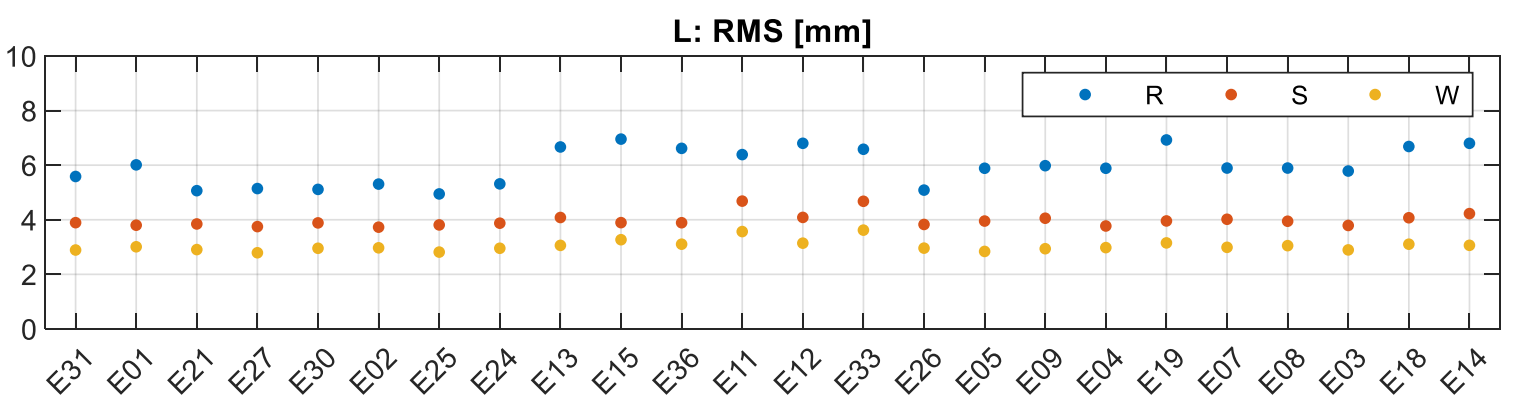

clock modelling applied

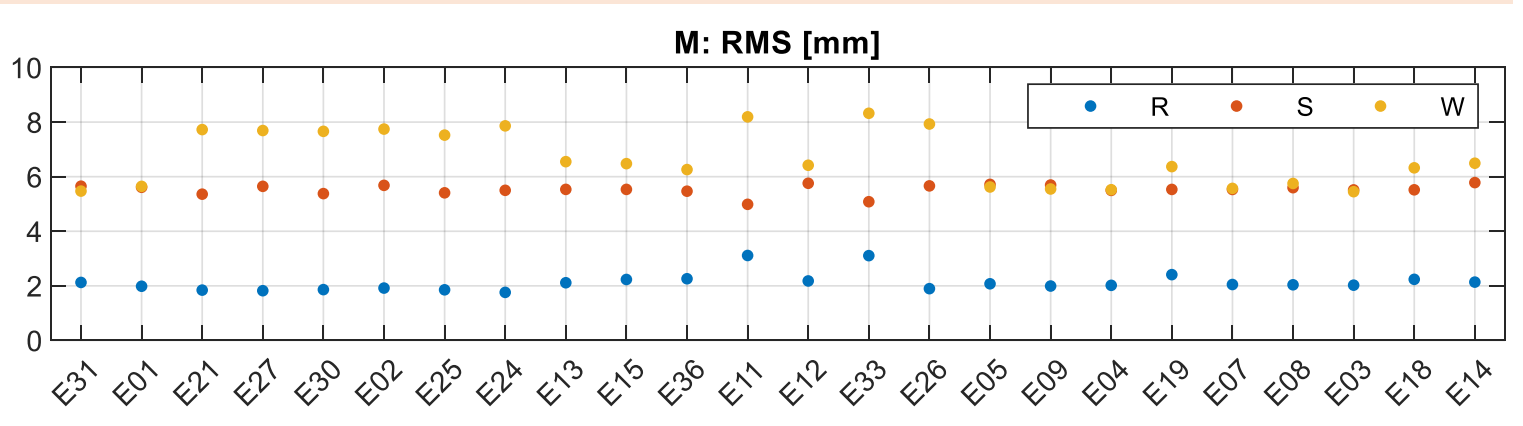

- ECOM2 cannot fully capture mismodelled NGP especially for low $\beta$ angles 


\section{Results - signatures of the NGP mismodelling}

station coordinates
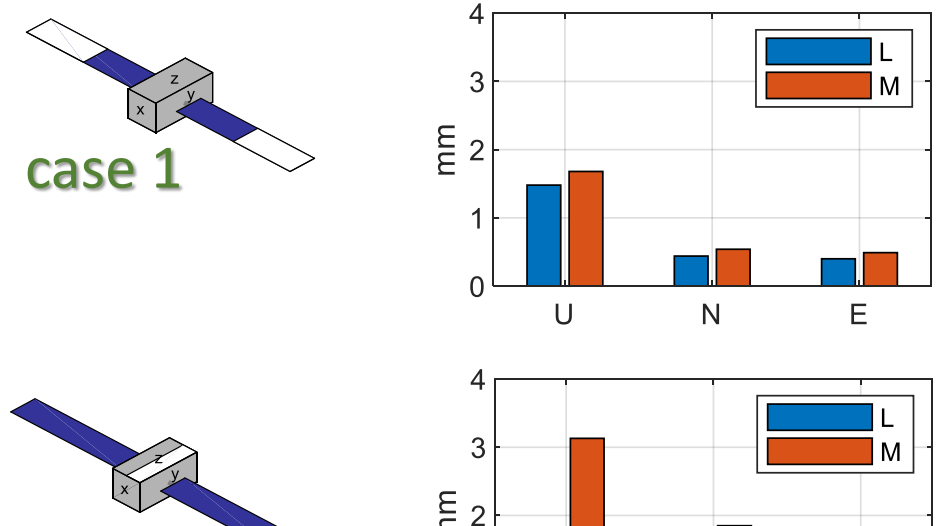

case 2
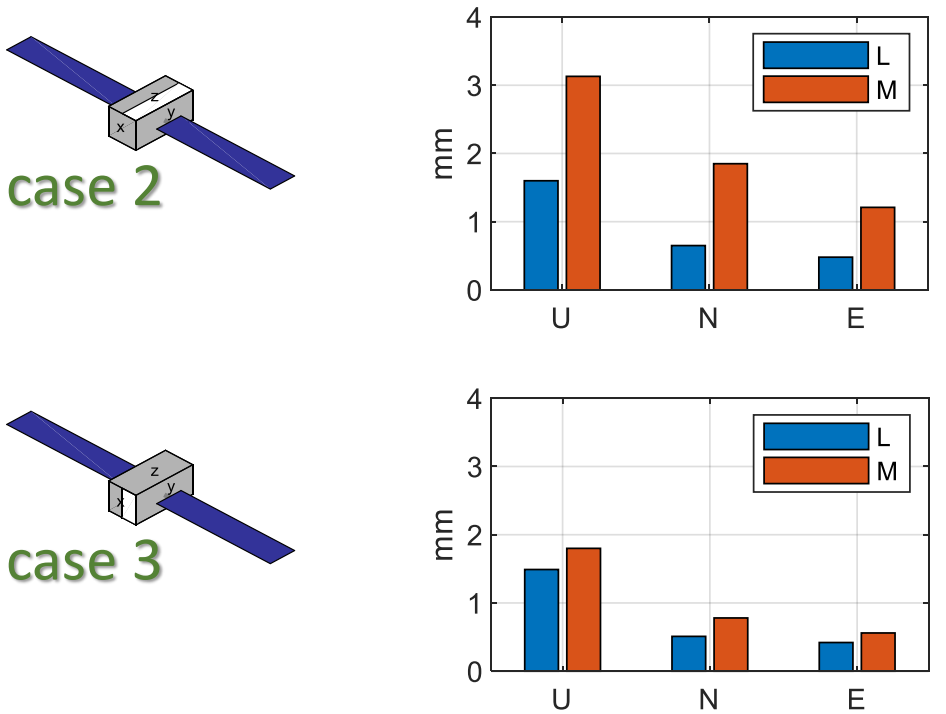

ECOM2
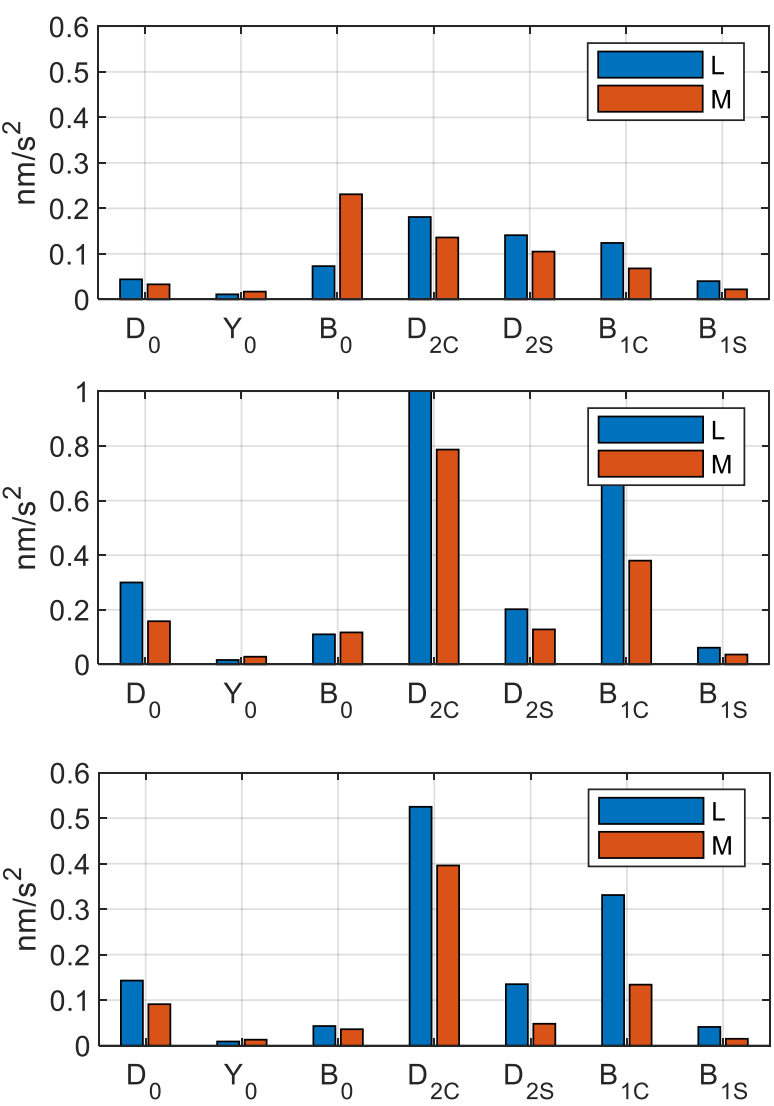
satellite clocks
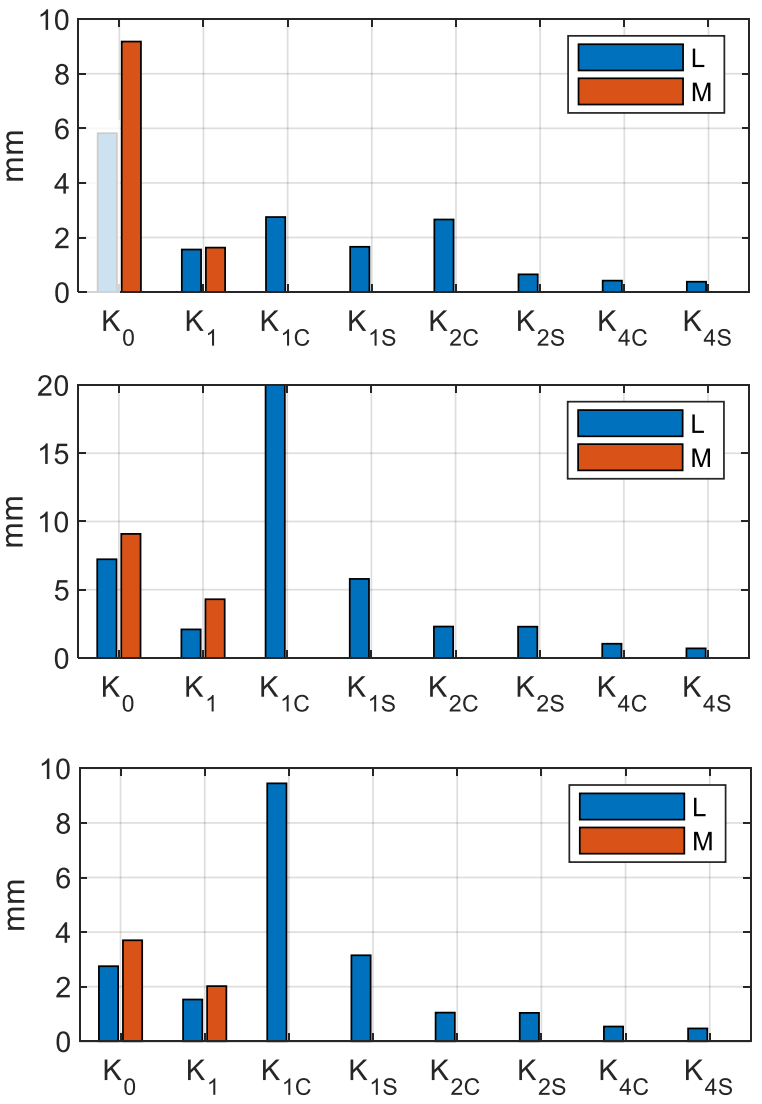

- signatures (values averaged over one year) are based on the solution with all ambiguities resolved

- signatures may give a hint regarding the possible origin of the mismodelled NGP

- another signatures are needed to clearly distinguish between case 2 and 3 


\section{Results - signatures of the NGP mismodelling (orbit)}

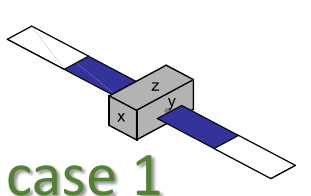

case 1

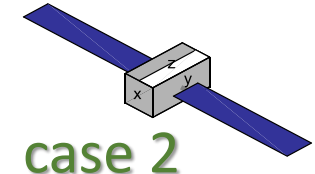

case 2

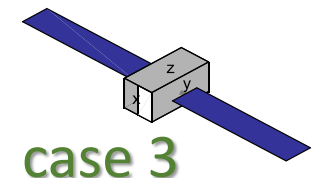

case 3

Note:

- satellites on elliptical orbits are not included in these (and subsequent) plots

- separate line is plotted for each orbital plane

- moving average filter with a $3^{\circ}$ window is applied to the data clock modelling applied $\operatorname{M:~} \operatorname{RMS}(\beta)[\mathrm{mm}]$
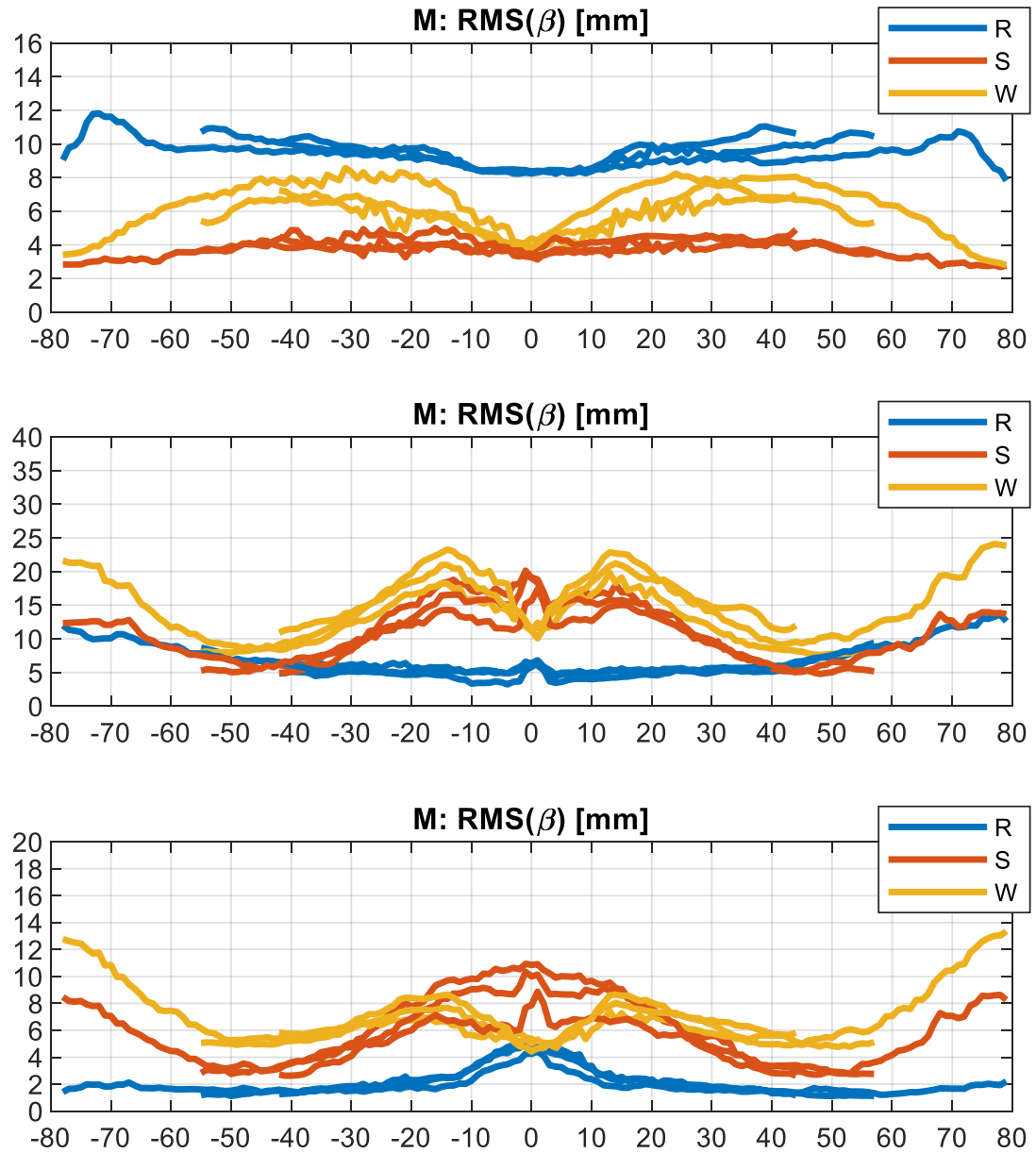


\section{Results - signatures of the NGP mismodelling (ECOM2)}

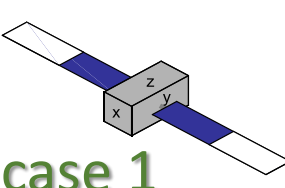

case 1
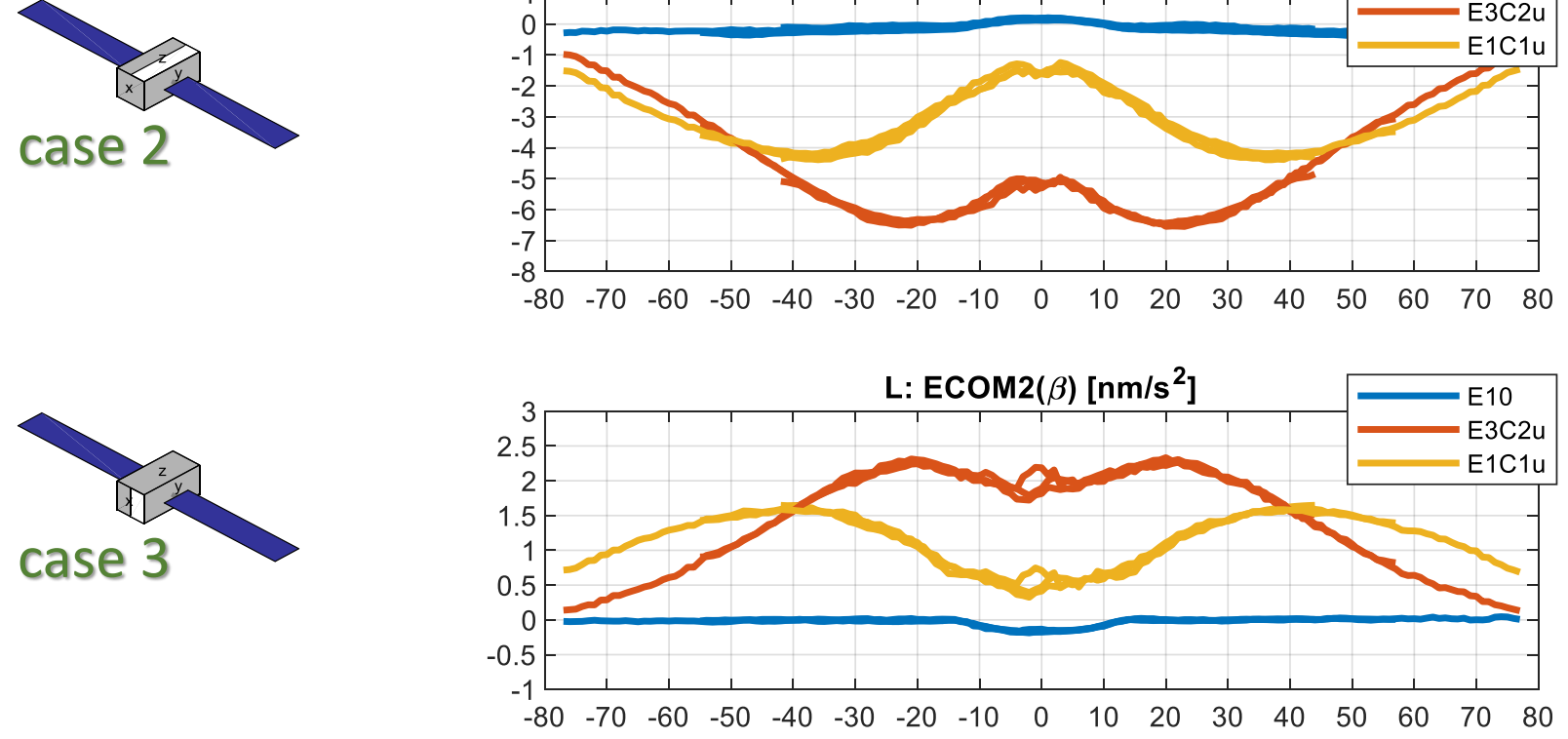

clock modelling applied

$\mathrm{M}: \operatorname{ECOM2}(\beta)\left[\mathrm{nm} / \mathrm{s}^{2}\right]$
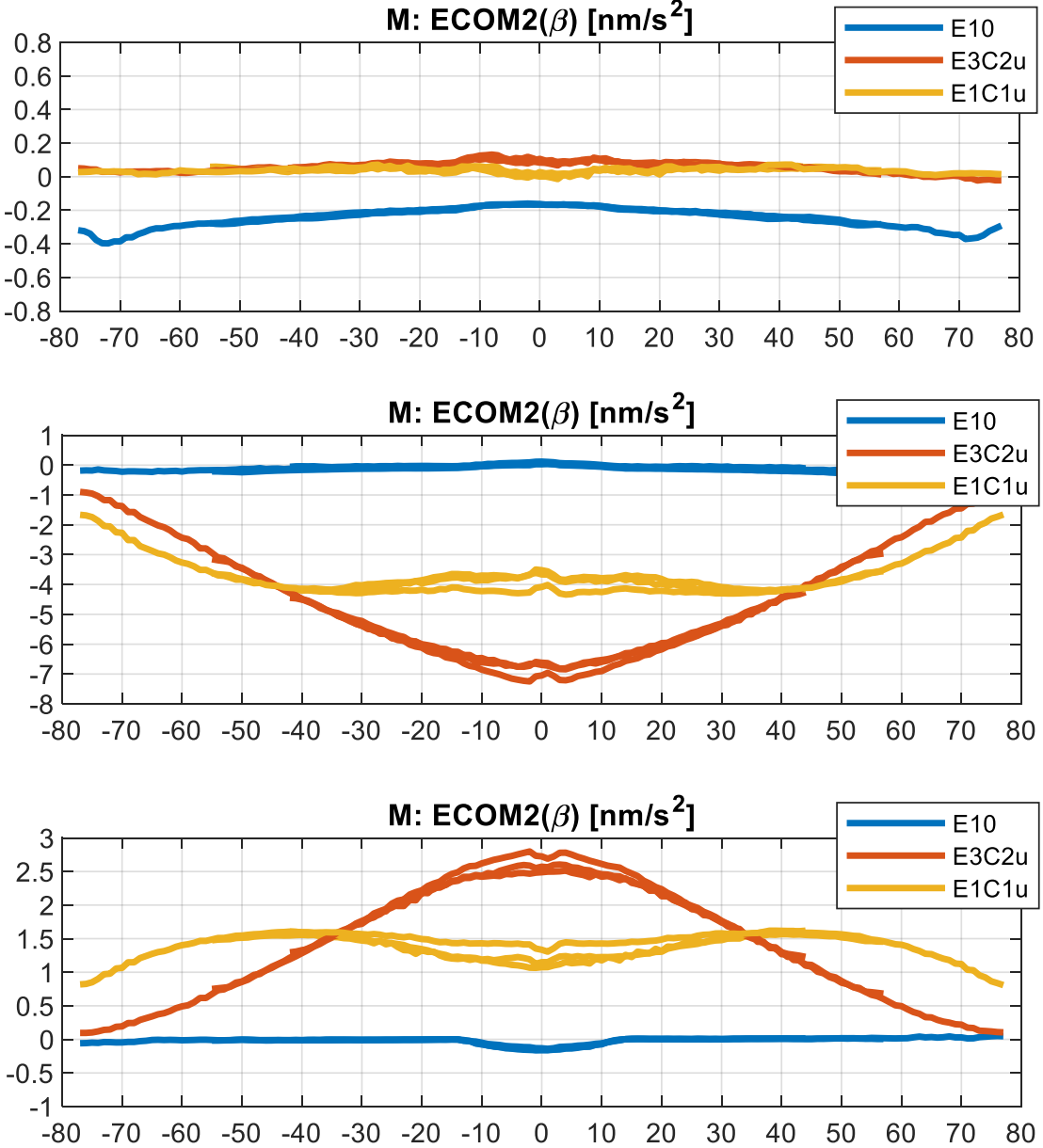

- clock modelling considerably influences the ECOM2 estimates

- signatures for all the cases are distinguishable 


\section{Results - signatures of the NGP mismodelling (satellite clocks)}
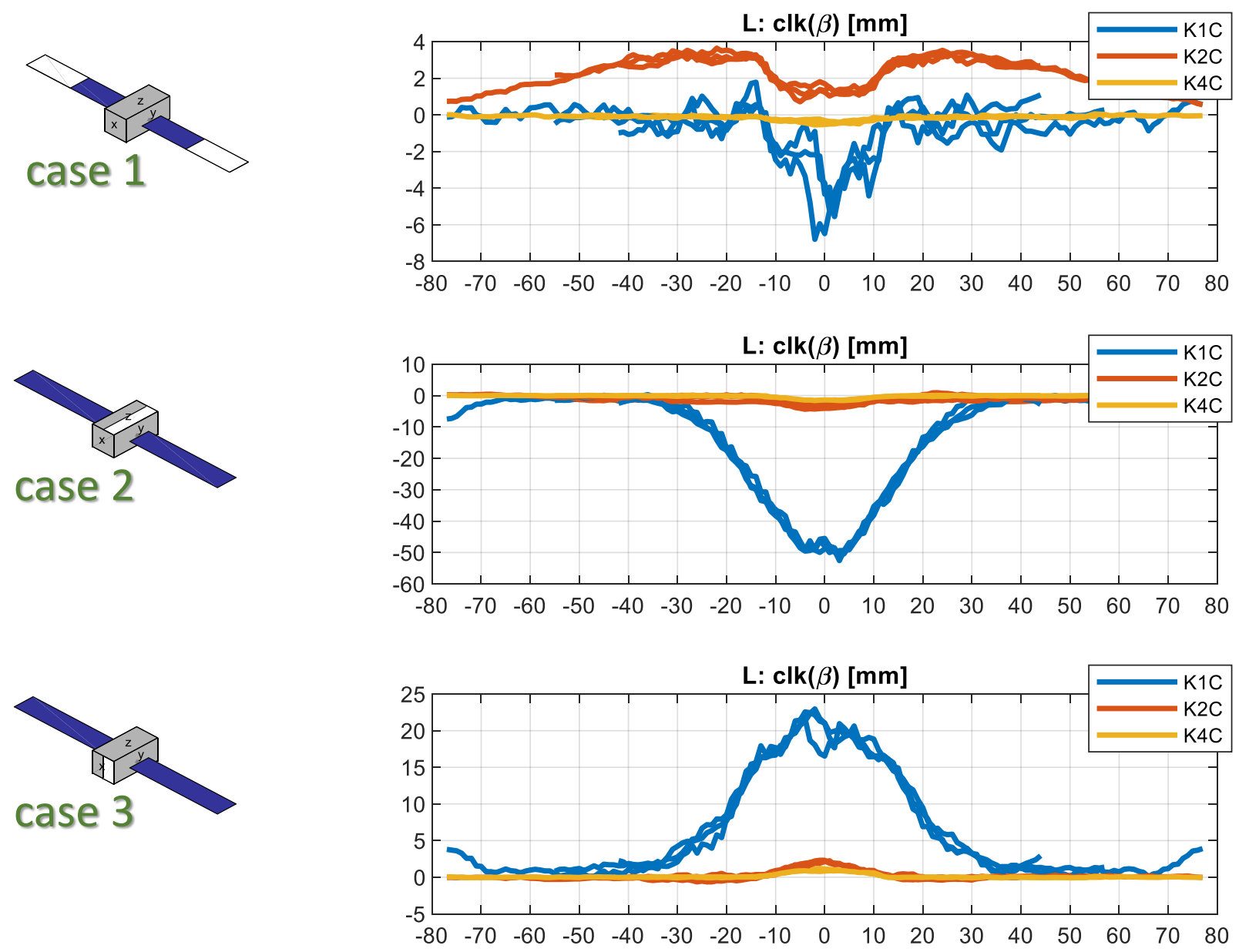

L: epoch-wise clocks 


\section{Summary}

- Deficiencies of the non-gravitational force models (here introduced by modification of the boxwing model) are only partially captured by ECOM2 parameters. The remaining parts affect satellite clock estimates and are also visible in satellite orbit and station coordinates.

- Careful analysis of some parameters (mainly ECOM2 and satellite clock corrections) can lead to valuable clues about a possible source of mismodeled NGPs.

- Estimation options considerably change the signatures of mismodelled NGPs. Signatures can be defined individually for particular estimation scenarios in the simulated environment.

- Clock modelling stabilizes the entire solution by reducing the number of estimated parameters. Unmodelled NGPs are then more visible in the orbits. 


\section{Thank you}


- how the results of precise orbit determination are affected by mismodelled non-gravitational forces

- how the potential exploitation of stable passive H-masers (e.g. by linear clock modelling) can influence the solution

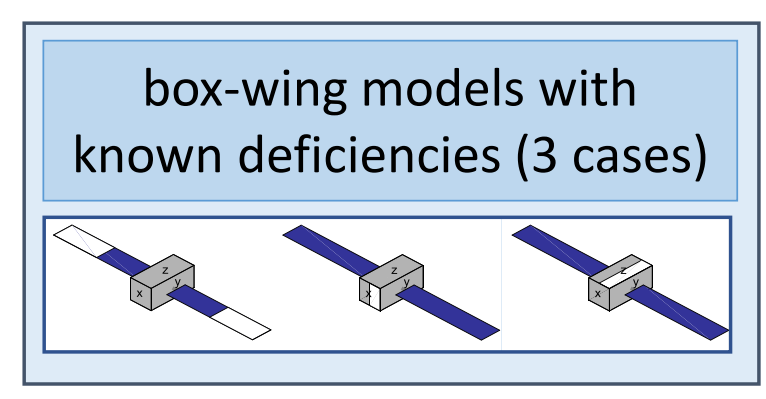

REFERENCE ("truth")

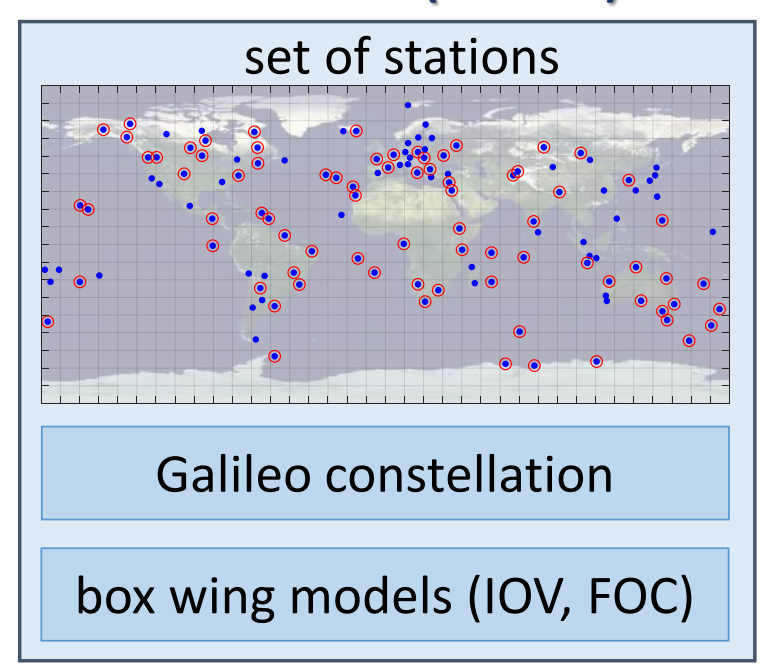

\section{Precise Orbit} Determination

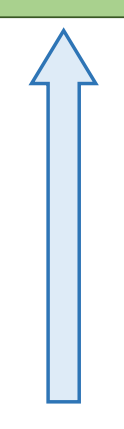

observation simulator (366 daily batches)

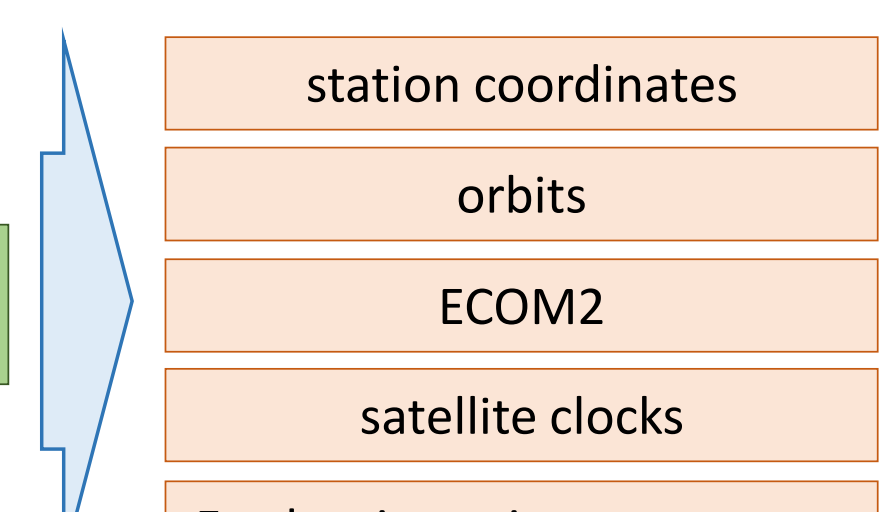

Earth orientation parameters

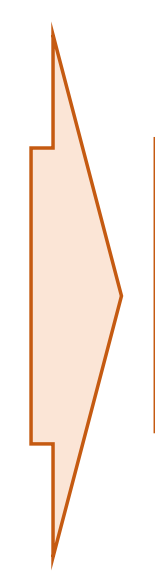

identification of characteristic signatures corresponding to the modified parameters of the box-wing model

- different deficiencies of the box-wing model lead to different characteristic signatures observed in the estimated parameters

- the clear signatures are mainly seen in ECOM2 parameters and satellite clock corrections

- clock modelling stabilizes the solution and also considerably changes the signatures of mismodelled NGPs 